\title{
An intramolecular theory of the mass-independent isotope effect for ozone. II. Numerical implementation at low pressures using a loose transition state
}

\author{
B. C. Hathorn and R. A. Marcus \\ Noyes Laboratories, 127-72, California Institute of Technology, Pasadena, California 91125
}

(Received 16 March 2000; accepted 8 September 2000)

\begin{abstract}
A theory is described for the variation in the rate constants for formation of different ozone isotopomers from oxygen atoms and molecules at low pressures. The theory is implemented using a simplified description which treats the transition state as loose. The two principal features of the theory are a phase space partitioning of the transition states of the two exit channels after formation of the energetic molecule and a small $(\mathrm{ca} .15 \%)$ decrease in the effective density of states, $\rho$ [a " "non-Rice-Ramsperger-Kassel-Marcus (RRKM) effect'], for the symmetric ozone isotopomers [B. C. Hathorn and R. A. Marcus, J. Chem. Phys. 111, 4087 (1999)]. This decrease is in addition to the usual statistical factor of 2 for symmetric molecules. Experimentally, the scrambled systems show a "mass-independent" effect for the enrichments $\delta$ (for trace) and $E$ (for heavily) enriched systems, but the ratios of the individual isotopomeric rate constants for unscrambled systems show a strongly mass-dependent behavior. The contrasting behavior of scrambled and unscrambled systems is described theoretically using a "phase space" partitioning factor. In scrambled systems an energetic asymmetric ozone isotopomer is accessed from both entrance channels and, as shown in paper I, the partitioning factor becomes unity throughout. In unscrambled systems, access to an asymmetric ozone is only from one entrance channel, and differences in zero-point energies and other properties, such as the centrifugal potential, determine the relative contributions (the partitioning factors) of the two exit channels to the lifetime of the resulting energetic ozone molecule. They are responsible for the large differences in individual recombination rate constants at low pressures. While the decrease in $\rho$ for symmetric systems is attributed to a small non-RRKM effect $\eta$, these calculated results are independent of the exact origin of the decrease. The calculated "mass-independent" enrichments, $\delta$ and $E$, in scrambled systems are relatively insensitive to the transition state (TS), because of the absence of the partitioning factor in their case (for a fixed non-RRKM $\eta$ ). They are compared with the data at room temperature. Calculated results for the ratios of individual isotopomeric rate constants for the strongly mass-independent behavior for unscrambled systems are quite sensitive to the nature of the TS because of the partitioning effect. The current data are available only at room temperature but the loose TS is valid only at low temperatures. Accordingly, the results calculated for the latter at $140 \mathrm{~K}$ represent a prediction, for any given $\eta$. At present, a comparison of the $140 \mathrm{~K}$ results can be made only with room temperature data. They show the same trends as, and are in fortuitous agreement, with the data. Work is in progress on a description appropriate for room temperature. (C) 2000 American Institute of Physics. [S0021-9606(00)01445-8]
\end{abstract}

\section{INTRODUCTION}

In a recent paper, hereafter called paper $\mathrm{I},{ }^{1}$ it was postulated that a small ( $c$. 15\%) non-Rice-Ramsperger-KasselMarcus (RRKM) effect gave rise to a dramatic effect, the so-called mass-independent isotope effect, in ozone formation. ${ }^{2-24}$ Recently, experiments on specific elementary reaction steps ${ }^{25-28}$ in contrast with scrambled systems, showed dramatic mass-dependent effects. This dichotomy between the behavior of individual recombination steps and of scrambled systems is treated in the present paper, using the formalism given in paper I. The focus there was the derivation of equations to treat these experiments. The field itself, it has been suggested, provides added insight into diverse phenomena, such as stratospheric/tropospheric mixing, stratospheric dynamics, oxidative processes in the strato- sphere and mesosphere, the evolution of an atmosphere like that of Mars and the ancient atmosphere on Earth. ${ }^{29-31}$

As we have noted in paper I, the difference in physical terms in this theory of the two types of experiments is that in the unscrambled experiments only one of the entrance channels to form a vibrationally excited ozone isotopomer, such as $\mathrm{XYY}^{*}$, is accessed, e.g., $\mathrm{X}+\mathrm{YY} \rightarrow \mathrm{XYY}{ }^{*}$. In scrambled experiments $\mathrm{XYY}^{*}$ is also formed via an additional channel, $\mathrm{Y}+\mathrm{XY} \rightarrow \mathrm{XYY} \mathrm{Y}^{*}$, since the isotopic exchange reaction $\mathrm{X}+\mathrm{YY} \rightleftharpoons \mathrm{XY}+\mathrm{Y}$ is extensive under scrambled conditions. A different and mass-specific percentage of the transition state phase space of the two exit channels, described by a "partitioning factor," is occupied when the access to it is from only one channel. This difference in conditions led in the kinetic scheme to a marked difference in the theoretical ex- 
pressions in paper I for the two types of experiments.

We describe in the present paper the numerical results arising from the theory of paper I and compare them with experiment. Theoretical expressions for several other quantities treated there are also calculated and compared with the data. As noted previously, to make the calculations we use RRKM theory, modified to include a small non-RRKM correction for the symmetric molecules. For the calculations described in the present paper, we employ in addition a simplified model of the transition state, a loose transition state, numerically for the density of states of the vibrationally excited molecule and the transition state sum of states. The enrichment results for scrambled systems, $\delta$ and $E$, are relatively insensitive to the nature of the TS and a comparison can be made directly with the body of the data, which are at room temperature. Results for the ratios of individual isotopomeric rate constants are, however, quite sensitive to the nature of the TS, because of the partitioning factor. Since the loose TS is valid only at low temperatures, the calculated results (here at $140 \mathrm{~K}$ ) for the individual rate constants of the unscrambled systems are compared with the room temperature data. They represent predictions for future low temperature data. Further numerical calculations using a model more appropriate for the negative temperature coefficient of the recombination and exchange rates is in progress.

As discussed in paper I, RRKM theory is used as the zeroth-order theory, with a small perturbation correction for the effective density of states of the symmetric isotopomeric ozone molecules, $\mathrm{XXX}$ and $\mathrm{XYX}$, as compared with the asymmetric ones YXX or YXZ. (This correction is apart from symmetry numbers, which are also included.) A rationale, in terms of sparsity of states and fewer overlapping resonances for the symmetric molecules, was described in paper I. The lifetime of the small and vibrationally excited ozone molecule, as estimated from the data, ${ }^{32}$ is of the order of 3 ps at room temperature, which is also in the neighborhood ( 0.1 to $1 \mathrm{ps})$ of the time scale expected for some nonRRKM behavior. The "non-RRKM" correction is small ( $c a$. $15 \%)$ relative to what such a correction could be, but is large in its consequence for the phenomena discussed in the present paper. Its importance is magnified in its effect on the enrichment factors $\delta$ and $E$ given later, and is such that it might be difficult to detect in usual thermal unimolecular reaction rate experiments.

Although we have suggested a non-RRKM origin for the reduced density of states of the symmetric molecules (a reduction apart from symmetry number effects), we note that the results described below are independent of the exact origin of the reduction. There are, also, further experimental consequences of the suggested origin, described later.

In Sec. II we recall the relevant equations, including those for the rate constants of individual reactions as well as an effective rate constant $k_{\text {rec }}^{\text {eff }}$ contributing to studies made with isotopically scrambled conditions (extensive isotopic exchange) and so contributing to the theoretical expression for $\delta$ or $E$. The expressions are used in Sec. III to obtain equations for the rate constants and for the enrichment. The numerical implementation of the expressions is described in Sec. IV, applied in Sec. V and discussed in Sec. VI.

\section{EQUATIONS FOR RATE CONSTANTS AND FOR ENRICHMENTS}

\section{A. Recombination rate constant at low pressures}

Most studies of the mass-independent isotope effect have been made in the low pressure region (three-body recombination region) and we focus on it in the present paper. However, to obtain more information on the transition state, we shall also refer to data for the recombination reaction at high pressures ${ }^{32}$ and to data on the isotopic exchange reaction. ${ }^{33-35}$

The low pressure formula for the rate constant $k_{\mathrm{rec}}^{0, a}$ for recombination in a reaction channel $a$ is, when there are two exit channels for redissociation of an energetic molecule, ${ }^{1}$

$$
\begin{aligned}
k_{\mathrm{rec}}^{0, a} & =\omega \sum_{J} \int_{E}\left[N_{a}^{\dagger} /\left(N_{a}^{\dagger}+N_{b}^{\dagger}\right)\right] \rho e^{-E / k_{B} T} d E / Q_{a} \\
& \equiv \frac{\omega}{Q_{a}} \sum_{J} \int_{E} Y_{a} \rho e^{-E / k_{B} T} d E,
\end{aligned}
$$

where $\omega$ is the deactivation collision frequency in the low pressure (low energy) region, $\rho(E, J)$ is the density of states of an energetic molecule at energy $E$ and total angular momentum quantum number $J, N_{a}^{\dagger}(E, J)$ is the number of quantum states in the transition state for entrance channel $a, N_{b}^{\dagger}(E, J)$ is that for the alternative exit channel $b$, and $Q_{a}$ is the partition function of the recombining pair in channel $a$ in their center of mass frame, and includes the electronic partition function. For $k_{\mathrm{rec}}^{0, b}$ the expression is the same as (2.1) but with $a$ and $b$ interchanged. The factor $Y_{a} \equiv N_{a}^{\dagger} /\left(N_{a}^{\dagger}\right.$ $\left.+N_{b}^{\dagger}\right)$ represents a "partitioning factor" between the two channels, and is defined to be zero unless at least one of the two channels is open for a given energy and angular momentum, i.e., $N_{a}^{\dagger}+N_{b}^{\dagger} \geqslant 1$.

The $\rho$ in Eq. (2.1) does not include a symmetry number. When the reaction is $\mathrm{X}+\mathrm{YX} \rightarrow \mathrm{XYX}^{*}$, where $\mathrm{Y}$ may or may not equal $\mathrm{X}$, the factor $N_{a}^{\dagger} /\left(N_{a}^{\dagger}+N_{b}^{\dagger}\right)$ becomes $1 / 2$ and the energetic molecule is symmetric. This factor of 2 can then be absorbed in $\rho$ which for this case now has a symmetry number of 2 .

\section{B. High pressure recombination rate constant}

The recombination rate constant $k_{\text {rec }}^{\infty, a}$ of a reaction in the high pressure region is

$$
k_{\mathrm{rec}}^{\infty, a}=\frac{1}{h Q_{a}} \sum_{J} \int_{E} N_{a}^{\dagger} e^{-E / k_{B} T} d E .
$$

For reactions such as $\mathrm{X}+\mathrm{YY} \rightarrow \mathrm{XYY}$ or $\mathrm{YXY}$, there is a separate rate constant for forming each reaction product. For reaction $\mathrm{X}+\mathrm{YZ} \rightarrow \mathrm{XYZ}$ when $\mathrm{YZ}$ is heteronuclear, when the transition state in the entrance channel is loose (freely rotating $\mathrm{YZ}$ in the transition state), the right-hand side of Eq. (2.2) for the high pressure rate constant should be multiplied by $1 / 2$, since only $1 / 2$ of the transition state species $(\mathrm{X}+\mathrm{YZ})^{\dagger}$ leads to $\mathrm{XYZ}^{*}$, the other half leading to the isomer $\mathrm{XZY}^{*}$. If the transition state for this heteronuclear $\mathrm{YZ}$ case is not loose, a weighting factor different from $1 / 2$ is needed. For example, if 1 and 2 denote the two reaction 
paths in the entrance channel, the $\rho$ for the vibrationally excited molecule obtained from entrance channel $a$ has a $\rho$ weighted by $N_{a, 1}^{\dagger} /\left(N_{a, 1}^{\dagger}+N_{a, 2}^{\dagger}\right)$ for reaction path $1, N_{a, i}^{\dagger}$ being the number of states for path $i$. When the transition state is loose, this factor reduces to $1 / 2$.

While this factor of $1 / 2$ is not explicitly cited in Eq. (2.2), it would be used when appropriate in applications. When there are two distinguishable isomeric products possible, the recombination rate constant $k_{\text {rec }}^{\infty}$ refers to forming a specific ozone isotopomer.

\section{Isotopic exchange rate constants}

Two exchange reactions are $\mathrm{X}+\mathrm{Y}_{2} \rightarrow \mathrm{XY}+\mathrm{Y}$ and $\mathrm{X}+\mathrm{YZ} \rightarrow \mathrm{XY}+\mathrm{Z}$ when $\mathrm{Z} \neq \mathrm{Y}, \mathrm{X}$. When the entrance channel is denoted by $a$ and the exit channel of this exchange reaction by $b$, the isotopic exchange rate constant was shown in paper I to be, at low pressures,

$$
k_{\mathrm{ex}}^{a}=\frac{1}{h Q_{a}} \sum_{J} \int_{E} \frac{N_{b}^{\dagger} N_{a}^{\dagger}}{N_{a}^{\dagger}+N_{b}^{\dagger}} e^{-E / k_{B} T} d E,
$$

where $N_{a}^{\dagger}$ and $N_{b}^{\dagger}$ again depend on $E$ and $J$. If the transition state is loose and if $\mathrm{Z} \neq \mathrm{Y}$, one again multiplies Eq. (2.3) by $1 / 2$. The $\omega$ does not appear in (2.3), and the equation assumes that there is no memory, other than the constants of the motion $E$ and $J$, of the state of the entrance channel in any subsequent dissociation of the molecule.

\section{Relation between $\boldsymbol{k}_{\mathrm{rec}}^{\infty, a}(\omega=\infty)$ and $\boldsymbol{k}_{\mathrm{ex}}^{a}(\omega \rightarrow 0)$}

As shown in paper I, the value of $k_{\text {rec }}^{\infty}$ for the reaction

$$
X+X_{2} \rightarrow X_{3} \quad(\omega \rightarrow \infty)
$$

can be inferred from the rate constant $k_{\mathrm{ex}}$ for the isotopic exchange reaction $(2.5)$,

$$
\mathrm{X}+\mathrm{Y}_{2} \rightarrow \mathrm{XY}+\mathrm{Y} \quad(\omega \rightarrow 0) .
$$

For example, when the former is unknown or very uncertain because of some complication occurring in the high pressure region where it is measured, ${ }^{32} k_{\text {rec }}^{\infty}$ can be approximately estimated from $k_{\mathrm{ex}}(\omega \rightarrow 0)$.

From Eqs. (2.2) and (2.3), neglecting differences between $N_{a}^{\dagger}$ and $N_{b}^{\dagger}$ in Eq. (2.3), we have

$$
k_{\mathrm{rec}}^{\infty} \approx 2 k_{\mathrm{ex}},
$$

where the $k_{\mathrm{ex}}$ is measured at $(\omega \rightarrow 0)$.

Equations (2.2) and (2.3) differ only in that $N_{a}^{\dagger}$ in one is replaced by $N_{a}^{\dagger} N_{b}^{\dagger} /\left(N_{a}^{\dagger}+N_{b}^{\dagger}\right)$ in the other. Neither integrand depends on a density of states of the ozone, for example. One can obtain a more accurate relation by evaluating the specific expressions for $k_{\text {rec }}^{\infty}$ and $k_{\mathrm{ex}}$, and we have done so in Sec. VE.

\section{E. Effective rate constants and enrichments in scrambled systems}

For a scrambled system at low pressures the isotopic exchange reactions, e.g.,

$$
\mathrm{X}+\mathrm{YZ} \rightleftharpoons \mathrm{Z}+\mathrm{XY}
$$

where $Z \neq X$, are extensive, and a local isotopic equilibrium between atomic and diatomic species is readily established. Reaction (2.7) occurs via a highly energetic intermediate, $\mathrm{XYZ}^{*}$, which can either dissociate to form products of (2.7) or the reactants, or more rarely be deactivated by collision to form XYZ. We denote the recombination rate constants to form this XYZ by $k_{\text {rec }}^{0, a}$ and $k_{\text {rec }}^{0, b}$, where $b$ is later defined as the channel having a diatomic reactant with the higher zeropoint energy, say XY,

$$
\begin{array}{cc}
\mathrm{X}+\mathrm{YZ} \rightarrow \mathrm{XYZ} & k_{\mathrm{rec}}^{0, a}, \\
\mathrm{Z}+\mathrm{XY} \rightarrow \mathrm{XYZ} & k_{\mathrm{rec}}^{0, b} .
\end{array}
$$

The rate of formation of XYZ under these conditions of rapid isotopic exchange is

$$
\frac{d \mathrm{XYZ}}{d t}=k_{\mathrm{rec}}^{0, a} \mathrm{X} \cdot \mathrm{YZ}+k_{\mathrm{rec}}^{0, b} \mathrm{Z} \cdot \mathrm{XY}=k_{\mathrm{rec}}^{\mathrm{eff}, a} \mathrm{X} \cdot \mathrm{YZ},
$$

where

$$
k_{\mathrm{rec}}^{\mathrm{eff}, a}=k_{\mathrm{rec}}^{0, a}+k_{\mathrm{rec}}^{0, b} K_{\mathrm{ex}}=k_{\mathrm{rec}}^{0, a}+k_{\mathrm{rec}}^{0, b} \frac{Q_{b}}{Q_{a}} .
$$

Here, $K_{\mathrm{ex}}$ is the equilibrium constant for reaction (2.7) and the $Q$ 's are conventional partition functions containing the zero-point energies. The combination of rate constants in (2.11) appears below, and in paper I, as $k_{6, q 6}^{a s}+k_{q, 66}^{a s} K_{\text {ex }}$ in an expression for the isotopic enrichment $\delta$, the superscript " $a s$ " denoting the formation of an asymmetric isotopomer. The theoretical expression for $k_{\mathrm{rec}}^{\mathrm{eff}, a}$ is simpler, it will be seen, than that for $k_{\mathrm{rec}}^{0, a}$ or $k_{\mathrm{rec}}^{0, b}$ alone, as was also noted in paper I. In particular, the "partitioning factor," $N_{a}^{\dagger} /\left(N_{a}^{\dagger}+N_{b}^{\dagger}\right)$, present in Eq. (2.1) is absent in the equation for $k_{\mathrm{rec}}^{\mathrm{eff}, a}$,

$$
k_{\mathrm{rec}}^{\mathrm{eff}, a}=\omega \sum_{J} \int_{E} \rho e^{-E / k_{B} T} d E / Q_{a} .
$$

For the $k_{\text {rec }}^{\text {eff, } b}$, we have similarly

$$
k_{\mathrm{rec}}^{\mathrm{eff}, b}=k_{\mathrm{rec}}^{0, b}+k_{\mathrm{rec}}^{0, a} / K_{\mathrm{ex}}=\omega \sum_{J} \int_{E} \rho e^{-E / k_{B} T} d E / Q_{b} .
$$

In each of these expressions, the domain of the integral and summation is restricted to the region where $N_{a}^{\dagger}+N_{b}^{\dagger} \geqslant 1$.

For the present paper we first consider the enrichment factor ${ }^{q} \delta$ where two of the three isotopes are present in trace amounts, the trace isotope being denoted by Q. We consider studies of the enrichment in these trace isotopes in the low pressure "three-body" region. We then consider the enrichments $E$ of the various isotopomers in heavily enriched systems.

In terms of rate constants and equilibrium constants of the reactions

$$
\begin{aligned}
& \mathrm{O}+\mathrm{O}_{2} \rightarrow \mathrm{O}_{3} \quad k_{6,66}^{s}, \\
& \mathrm{O}+\mathrm{QO} \rightarrow \mathrm{QOO} \quad k_{6, q 6}^{a s}, \\
& \mathrm{O}+\mathrm{QO} \rightarrow \mathrm{OQO} \quad k_{6, q 6}^{s}, \\
& Q+\mathrm{O}_{2} \rightarrow \mathrm{QOO} \quad k_{q, 66}^{a s},
\end{aligned}
$$




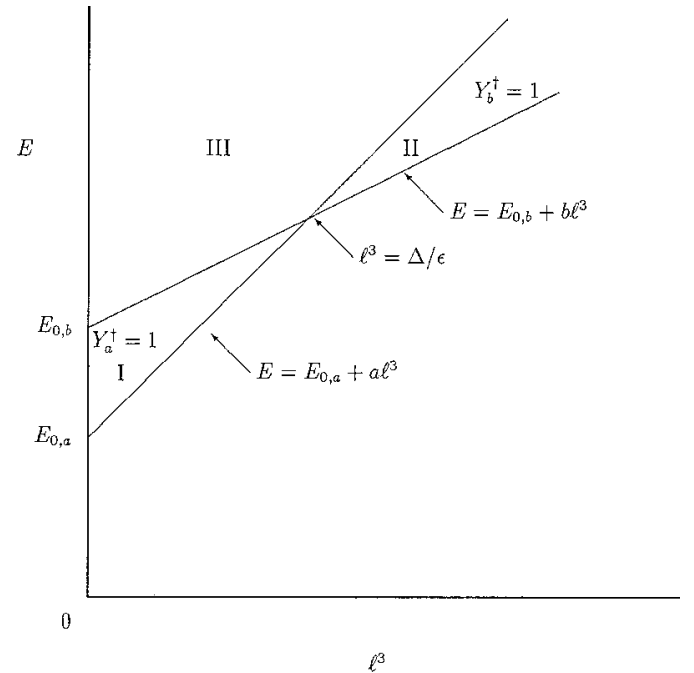

FIG. 1. Description of the contributions I, II, and III to the integration region for $k_{\mathrm{rec}}^{0}$ in $\left(E, \ell^{3}\right)$ space, for the case $E_{0, b} \geqslant E_{0, a}$ and $a \geqslant b$.

$$
\mathrm{O}+\mathrm{QO} \rightleftharpoons \mathrm{Q}+\mathrm{O}_{2} \quad\left(K_{\mathrm{ex}}\right),
$$

the rate of formation of QOO, using Eq. (2.11) above, is $\left(k_{6, q 6}^{a s}+k_{q, 66}^{a s} K_{\mathrm{ex}}\right) \mathrm{O} \cdot \mathrm{QO}$, where $K_{\mathrm{ex}}$ is the equilibrium constant for reaction (2.18). The rate of formation of OQO is $k_{6, q 6}^{s} \mathrm{O} \cdot \mathrm{QO}$, the $s$ denoting the formation of a symmetric molecule. The net rate of formation of an ozone molecule containing a $\mathrm{Q}$ is thus $\left(k_{6, q 6}^{a s}+k_{q, 66}^{a s} K_{\mathrm{ex}}+k_{6, q 6}^{s}\right) \mathrm{O} \cdot \mathrm{QO}$. Since the rate of formation of $\mathrm{O}_{3}$ is $k_{6,66}^{s} \mathrm{O} \cdot \mathrm{O}_{2}$, the ratio of reaction products formed (QOO+OQO) $/ \mathrm{O}_{3}$ is $\left[\left(k_{6, q 6}^{a s}+k_{q, 66}^{a s} K_{\mathrm{ex}}\right.\right.$ $\left.\left.+k_{6, q 6}^{s}\right) / k_{6,66}^{s}\right]\left(\mathrm{QO} / \mathrm{O}_{2}\right)$. The enrichment factor ${ }^{q} \delta$ is defined as the $\mathrm{QO} / \mathrm{O}_{2}$ ratio in the products divided by that in the oxygen reactant, minus one, and so, using Eq. (2.12) or (2.13),

$$
\begin{aligned}
{ }^{q} \delta & =\frac{2\left(k_{6, q 6}^{a s}+k_{q, 66}^{a s} K_{\mathrm{ex}}+k_{6, q 6}^{s}\right)}{3 k_{6,66}^{s}}-1 \\
& =\frac{2}{3} \frac{\sum_{J} \int\left(\rho_{q 66}^{a s}+\rho_{6 q 6}^{s}\right) e^{-E / k_{B} T} d E}{\sum_{J} \int \rho_{666}^{s} e^{-E / k_{B} T} d E}-1,
\end{aligned}
$$

where $\rho_{66 q}^{a s}$ is the density of states of the asymmetric molecule, ${ }^{16} \mathrm{O}^{16} \mathrm{O}^{q} \mathrm{O}, \rho_{6 q 6}^{s}$ is that for the symmetric molecule, ${ }^{16} \mathrm{O}^{q} \mathrm{O}^{16} \mathrm{O}$, and $\rho_{666}^{s}$ is the density of states for the ${ }^{16} \mathrm{O}^{16} \mathrm{O}^{16} \mathrm{O}$ molecule, which can only be symmetric. Here, $q=17$ or 18 . There are clearly no partitioning factors in (2.19b). In (2.19a)-(2.19b) we have set the various deactivation collision frequencies, $\omega$, equal to each other for notational brevity. However, in the equations we actually use, this approximation is not made. As in Eq. (2.19b), this quantity ${ }^{q} \delta$ was expressed in Eq. (2.20) of paper I as a sum over $J$ and an integral over $E$ in the $(J, E)$ integration domain where either $N_{a}^{\dagger} \geqslant 1$ or $N_{b}^{\dagger} \geqslant 1$.

Turning next to the enrichments $E_{i j j}, E_{j j j}$, and $E_{i j k}$ in paper I, we note that the integration in (2.12) and (2.13) is over a region where $N_{a}^{\dagger} \geqslant 1$ or $N_{b}^{\dagger} \geqslant 1$ or both. In the loose transition state approximation this integration region consists of I, II, and III in Fig. 1. A particular case of (2.11) is the $k_{6, q 6}^{a s}+k_{q, 66}^{a s} K_{\text {ex }}$ in Eq. (2.19a) for ${ }^{q} \delta$, the $6+q 6$ being the $a$ channel, since oxygen $q 6$ has a lower zero-point energy than 66.

For $E_{i j j}$ in general, Eqs. (4.18a) and (4.18b) of paper I can be combined to yield

$$
E_{i j j}=\frac{\left(k_{j, i j}^{s}+k_{\mathrm{rec}}^{\mathrm{eff}, c}\right)}{3 k_{6,66}^{s}} K_{6, j j}^{i, 6 j} \sigma-1 \quad(j \neq 6),
$$

where $c$ denotes $a$ or $b$, according to whether the $j+i j$ channel is an $a$ or $b$ channel. The $\sigma$ is 1 or 2 , according as $j$ $\neq 6$ or $j=6$, respectively. $K_{6, j j}^{j, 6 j}$ is the equilibrium constant for reaction (2.5), when $\mathrm{X}={ }^{6} \mathrm{O}$ and $\mathrm{Y}={ }^{j} \mathrm{O} . E_{j j j}$, given by Eq. (4.21) in paper $I$, is

$$
E_{j j j}=\frac{k_{j, j j}^{s} K_{6, j j}^{j, 6 j}}{2 k_{6,66}^{s}}-1=\frac{\omega}{Q_{a} k_{6,66}^{s}} \sum_{J} \int_{E} \rho e^{-E / k_{B} T} d E-1,
$$

where $\omega, \rho$, and $Q$ are the relevant quantities for the $j+j j$ recombination reaction.

$E_{i j k}$ for unequal $i, j$, and $k$ is given in paper I by Eq. (4.26), where each $k^{a s}$ now denotes the total formation rate constant for the sum of the two isotopomeric ozones. It is also given by Eq. (4.34) there as a sum and an integral over $(J, E)$ space. Once again, the partitioning factor becomes unity throughout.

\section{IMPLEMENTATION WHEN A LOOSE TRANSITION STATE IS ASSUMED}

When a loose transition state is assumed for simplicity (e.g., as in "phase space theory") the orbital angular momentum quantum number $\ell$ of the colliding reactants, the rotational angular momentum quantum number $j$ of the diatomic reactant, and the vibrational quantum number $v$ of the diatomic reactant, are good quantum numbers in the region of the transition state in the entrance channel $a$. When $k_{\text {rec }}^{\infty, a}$ is calculated using Eq. (2.2) and a loose transition state is assumed, it is convenient to use $j$ and $\ell$ for the angular momentum quantum numbers in the sum, instead of introducing the total (rotational plus orbital) angular momentum quantum number $J .^{36-39}$ However, when there are either two channels involved in the process, as in Eq. (2.3) for $k_{\mathrm{ex}}$, or when the density of states of the vibrationally excited intermediate plays a role, as in Eq. (2.1) for $k_{\mathrm{rec}}^{0, a}$, it is necessary to introduce the total angular momentum quantum number, $J$. In Eq. (2.3) it is assumed that there is no memory of the $(j, \ell)$ values in the initial channel $a$ by the outgoing $(j, \ell)$ 's in channel $b$, subject to the constraints of constant $E$ and $J$ (and, conceivably, some other quantum number). In this case the sum over $j, \ell, m_{j}$, and $m_{\ell}$ is replaced by a sum over $j, \ell, J$, and $M_{J}$. The sum over $M_{J}$ yields $(2 J+1)$ as a factor. ${ }^{40}$

For a loose transition state the effective potential for the relative motion of the colliding pair is the sum of an attractive potential, written often as $-C_{6} / r^{6}, r$ being the separation distance (atom to center of mass of the diatomic molecule), and a centrifugal potential, $\ell(\ell+1) \hbar^{2} / 2 \mu r^{2} \cdot{ }^{36-38}$ The energy barrier is found, as usual, by locating the $r$ where 
this sum is a maximum (the bottleneck to the reaction). The energy barrier $E^{\dagger}, a$ for channel $a$ and that for $b$ are then found to be $\mathrm{b}^{36-38,41-43}$

$$
E_{\ell, a}^{\dagger}=\frac{1}{3} a[\ell(\ell+1)]^{3 / 2}, \quad E_{\ell, b}^{\dagger}=\frac{1}{3} b[\ell(\ell+1)]^{3 / 2},
$$

and where

$$
\frac{1}{a}=\left(6 C_{6} \mu_{a}^{3}\right)^{1 / 2} / \hbar^{3}, \quad \frac{1}{b}=\left(6 C_{6} \mu_{b}^{3}\right)^{1 / 2} / \hbar^{3} .
$$

To calculate $N_{a}^{\dagger}$ the sum at a given $J$ and $E$ is made over all the $j, \ell$ and vibrational quantum numbers, $v$ which satisfy the energy condition

$$
E \geqslant E_{j, a}+E_{\ell, a}^{\dagger}+E_{0, a}+E_{v, a},
$$

where $E_{j, a}$ is the rotational energy of the diatomic molecule, $j(j+1) B_{D, a}, B_{D, a}$ being the rotational constant $\hbar^{2} / 2 I_{D, a}$ of the diatomic molecule in channel $a$. The third and fourth terms on the right-hand side of (3.2) denote the zero-point energy and the additional vibrational energy of the diatomic molecule in channel $a$. A second condition on the expression for $N_{a}^{\dagger}$ is that $j, \ell$, and $J$ satisfy the triangle inequality, ${ }^{36}$ $|j-\ell| \leqslant J \leqslant j+\ell$. Thereby, we can write

$$
\begin{aligned}
N_{a}^{\dagger}(E, J)= & \sum_{j=0}^{\infty} \sum_{\ell=|J-j|}^{J+j}(2 J+1) h\left(E-E_{j, a}-E_{\ell, a}^{\dagger}\right. \\
& \left.-E_{0, a}^{\dagger}\right) / \sigma_{D, a},
\end{aligned}
$$

where $h(x)=0$ for $x<0$ and $=1$ for $x>0$ and where in the present case $E_{v, a}$ in (3.2) equals zero to an excellent approximation at room or low temperatures, since $k_{B} T \ll h \nu$. The $\sigma_{D, a}$ is the symmetry number of the diatomic molecule in the transition state for $N_{a}^{\dagger}$. For $N_{b}^{\dagger}(E, J)$ the $a$ 's are replaced by b's.

For the evaluation of $k_{\text {rec }}^{0, a}$ using Eq. (2.1) it is necessary, in addition, to calculate $\rho$. For this purpose the triatomic molecule will be treated here as a "near-symmetric" top for simplicity of presentation. Its rotational energy $E_{\text {rot }}$ is then given by

$$
E_{\mathrm{rot}}=B_{A} J(J+1)+\left(B_{C}-B_{A}\right) K^{2},
$$

where $K \leqslant J$ ( $K$ is the projection of $J$ along the body fixed $z$ axis), $B_{A}$ and $B_{C}$ are the rotational constants $\hbar^{2} / 2 I_{A}$ and $\hbar^{2} I_{C} . I_{C}$ is the smallest moment of inertia of ozone. For $I_{A}$ we use the geometric mean of the other two moments of inertia. $^{39}$

The quantum number $K$ is either treated adiabatically or as "active." In the former case some particular correlation to the initial $(j, \ell)$ must be postulated. In this case the $N^{\dagger}$ 's and $\rho$ 's become functions of $K$ also. In contrast, in the $K$ active case, the $\rho$ is calculated by convolution of the number of $K$ states and the vibrational density of states, the limits on $|K|$ being the lessor of $J$ and the maximum allowed by the energy condition (3.2). We denote this value of $K$ by $K_{\max }$. That is, $K_{\max }$ is the lesser of

$$
\begin{aligned}
& K_{\max }=J, \\
& \left(B_{C}-B_{A}\right) K_{\max }^{2}=E-B_{A} J(J+1)+D-E_{\mathrm{vib}} .
\end{aligned}
$$

Here, $D$ is the electronic dissociation energy of the triatomic molecule and $E_{\text {vib }}$ is the vibrational energy of that molecule in excess of the zero-point energy. For any given $j$ and $\ell$ in the entrance channel, $E$ itself can be no less than the value given in Eq. (3.2), the excess being the initial translational energy in the center-of-mass system.

The value of $k_{\text {rec }}^{\infty}$ for a reaction (2.4), i.e., $\mathrm{X}+\mathrm{X}_{2} \rightarrow \mathrm{X}_{3}$, is given by $37,38,41-43$

$$
k_{\mathrm{rec}}^{\infty}=2(2 \pi / \mu)^{1 / 2}\left(2 C_{6}\right)^{1 / 3}\left(k_{B} T\right)^{1 / 6} \Gamma(2 / 3) / Q_{\mathrm{el}},
$$

where $\Gamma$ denotes the usual gamma function, and $Q_{\text {el }}$ represents the electronic partition function of the $\mathrm{O}\left({ }^{3} P_{2}\right)$ $+\mathrm{O}_{2}\left({ }^{3} \Sigma_{g}\right)$ pair, calculated from the energies and degeneracies of the individual electronic energy levels. ${ }^{44}$ This result is obtained by using classical partition functions for the transition state region and molecular fragments, when only collisions leading to the ground electronic state are reactive. Although not used directly in the present calculation, this analytic formula provides a useful check on the numerical calculations, agreeing to within $1 \%$, when applied to the homonuclear $\mathrm{X}+\mathrm{X}_{2} \rightarrow \mathrm{X}_{3}$ reactions.

\section{NUMERICAL IMPLEMENTATION}

Three critical quantities are involved in the implementation of the formulas for the rate constants, the sums and densities of states, the partition functions for the collision pair (which also arises in the calculation of the equilibrium constants for the exchange reaction) and the collision frequencies. The methods by which these quantities were obtained are outlined below.

\section{A. Sums and densities of states}

Molecular densities of states were determined numerically, making use of densities of states determined for noninteracting modes, and using a convolution to determine a joint density of states, ${ }^{38,39,45,46}$

$$
\rho_{1,2}(E)=\int_{0}^{E} \rho_{1}\left(E^{\prime}\right) \rho_{2}\left(E-E^{\prime}\right) d E^{\prime} .
$$

In order to accomplish this numerically, the grain size $\Delta E$ $=d E$ was varied until suitable convergence was obtained, in the present case a grain size of $\Delta E=1 \mathrm{~cm}^{-1}$ produced convergence of the numerically calculated PST value to the value which is obtained by a classical evaluation of the high pressure rate constant. Under this procedure, a density of states for a single mode is expressed as a vector, $\rho(E)$, the components of which are the number of states within the interval $(E, E+\delta E)$. A grain size smaller than $\approx 1 \mathrm{~cm}^{-1}$ would not be meaningful, for a molecule with a lifetime of 3 ps, without invoking uncertainty principle-broadened energy levels of the molecule and a discussion of isolated vs overlapping resonances. ${ }^{47}$

Vibrational densities of states are obtained numerically by a direct count of the states. For the vibrational states, anharmonicities, are not included, and the spectrum for a single mode is given by

$$
E_{n}=\left(n+\frac{1}{2}\right) h \nu .
$$


The implementation of the convolution integral (4.1) is performed via a Beyer-Swinehart algorithm. ${ }^{39,48}$

The rotational density of states for ozone molecules has been obtained by treating the molecule as a "near-symmetric top, , 39

$$
E(J, K)=\sqrt{B C} J(J+1)+(A-\sqrt{B C}) K^{2},
$$

where $A, B$, and $C$ are the molecular rotational constants. Each $(J, K)$ energy level appears with a degeneracy of $(2 J$ $+1) / \sigma$, where $\sigma$ is the usual symmetry number and is subject to the usual restriction $-J \leqslant K \leqslant J$. Since the ozone molecules of interest are on the ground ${ }^{1} A_{1}$ potential surface, no convolution with an electronic degeneracy is necessary to obtain the total density of states.

For the transition state, the angular momentum conserved states for channels $a$ and $b$ are obtained by a direct enumeration of the energy states in each of the two channels,

$$
\begin{aligned}
& E_{a}(j, \ell)=\frac{1}{3} a[\ell(\ell+1)]^{3 / 2}+B_{a} j(j+1), \\
& E_{b}(j, \ell)=\frac{1}{3} b[\ell(\ell+1)]^{3 / 2}+B_{b} j(j+1) .
\end{aligned}
$$

Each state being assigned a degeneracy of $(2 J+1)$ and being restricted by conservation of total angular momentum, $36,37,40$

$$
|J-\ell| \leqslant j \leqslant J+\ell .
$$

The value of $C_{6}$ for the atom-diatom interaction is taken to be half that of the $\mathrm{O}_{2}-\mathrm{O}_{2}$ collision pair, ${ }^{49} 2.78$ $\times 10^{-78} \mathrm{Jm}^{6}$. The results are not strongly dependent on the exact value of this constant, as in the high pressure limit, the dependence of the rate constant scales with $C_{6}^{1 / 3}$. The vibrational density of states is obtained, as in the case of the molecule, by a Beyer-Swinehart algorithm, with the exception that in the present case the lowest harmonic energy level is given by $D+\frac{1}{2} h \nu$, to account for the electronic dissociation energy. The total density of states is obtained by a convolution integral (4.1), and the sum of states for the respective channels is obtained from the integration over the respective densities of states,

$$
N(E, J)=\int \rho(E, J) d E .
$$

As previously noted, an additional factor of $1 / 2$ must be included, in the case of a homonuclear fragment, to account for the symmetry number of the diatomic fragment, but also in the case of the heteronuclear fragment, where half of the phase space represented by the sum of states is correlated to each of the two possible products in the recombination.

\section{B. Partition functions}

The partition functions for the atom-diatom pairs are given by a product of rotational, vibration, electronic, and translational partition functions, ${ }^{44}$

$$
Q_{(1,2)}=Q_{\text {rot }} Q_{\text {vib }} Q_{\text {elec }} Q_{\text {trans }} .
$$

The rotational and translational partition functions are given by their classical values ${ }^{44}$

$$
\begin{aligned}
& Q_{\text {rot }}=\frac{k T}{\sigma B}, \\
& Q_{\text {trans }}=\left(\frac{2 \pi \mu k T}{h^{2}}\right)^{3 / 2},
\end{aligned}
$$

where $\sigma$ is the usual symmetry number, $B$ is the rotational constant, and $\mu$ is the reduced mass of the atom-diatom pair.

The electronic and vibrational components of the partition function are calculated using the quantum partition functions ${ }^{44}$

$$
\begin{aligned}
& Q_{\mathrm{vib}}=\frac{\exp (-h \nu / 2 k T)}{1-\exp (-h \nu / k T)}, \\
& Q_{\text {elec }}=15+9 \exp (-158.5 / k T)+3 \exp (-226.5 / k T),
\end{aligned}
$$

where $\nu$ is the vibrational frequency of the diatomic molecule, and the energies appearing in the electronic partition function are in wavenumbers. ${ }^{44}$

\section{Collision frequencies}

We have assumed that the deactivating collision between a vibrationally excited ozone molecule and a bath gas molecule is governed by a Lennard-Jones-type potential, ${ }^{49}$

$$
V_{\mathrm{LJ}}(r)=4 \epsilon\left[\left(\frac{\sigma}{r}\right)^{12}-\left(\frac{\sigma}{r}\right)^{6}\right],
$$

where $\sigma$ and $\epsilon$ are standard interaction parameters for which we have assumed literature values, $\sigma=3.889 \AA$ and $\epsilon$ $=107.3 \mathrm{~K}^{50}$ The collision frequency is then given by ${ }^{39}$

$$
\begin{array}{r}
\omega=4.87 \times 10^{14} \sqrt{\frac{T}{1000}} \sqrt{\frac{20}{\mu_{\mathrm{O}_{3}, \mathrm{~N}_{2}}}} \\
\times\left(\frac{\sigma}{5}\right)^{2} \Omega_{2,2}^{*} \mathrm{~cm}^{3} \text { mole }^{-1} \mathrm{~s}^{-1} .
\end{array}
$$

The quantity $\mu$ is the reduced mass of the ozone-nitrogen pair in amu, which depends on the mass of the isotopes involved, $T$ is the absolute temperature, and the collision integral $\Omega_{2,2}^{*}$ is approximately given by ${ }^{39,51}$

$$
\begin{aligned}
\Omega_{2,2}^{*}= & 1.16145\left(T^{*}\right)^{-0.14874}+0.52487 e^{-0.7732 T^{*}} \\
& +2.16178 e^{-2.437887 T^{*}},
\end{aligned}
$$

where $T^{*}$ is the reduced temperature,

$$
T^{*}=\frac{T}{\epsilon} .
$$

We also note that in the ratios of third-order rate constants required for the calculation of enrichments, the factors in the collision frequency cancel with the exception of the square root of the ratio of the reduced masses [as in Eq. (5.1) below]. This result is consistent with the experimental observation (Ref. 28) that the ratios of rate constants have a negligible dependence on the nature of the third-body molecule even though the absolute values of the recombination rate constants have varied significantly. 
TABLE I. Estimated vibrational frequencies for ozone isotopomers.

\begin{tabular}{cccc}
\hline \hline${ }^{\mathrm{I} s o t o p o m e r}$ & $\nu_{1}$ & $\nu_{2}$ & $\nu_{3}$ \\
\hline${ }^{16} \mathrm{O}^{16} \mathrm{O}^{16} \mathrm{O}$ & 1104 & 701 & 1043 \\
${ }^{17} \mathrm{O}^{17} \mathrm{O}^{17} \mathrm{O}$ & 1071 & 680 & 1012 \\
${ }^{18} \mathrm{O}^{18} \mathrm{O}^{18} \mathrm{O}$ & 1041 & 661 & 984 \\
${ }^{16} \mathrm{O}^{16} \mathrm{O}^{17} \mathrm{O}$ & 1096 & 693 & 1037 \\
${ }^{16} \mathrm{O}^{17} \mathrm{O}^{16} \mathrm{O}$ & 1088 & 697 & 1025 \\
${ }^{16} \mathrm{O}^{16} \mathrm{O}^{18} \mathrm{O}$ & 1088 & 684 & 1032 \\
${ }^{16} \mathrm{O}^{18} \mathrm{O}^{16} \mathrm{O}$ & 1074 & 693 & 1008 \\
${ }^{17} \mathrm{O}^{17} \mathrm{O}^{16} \mathrm{O}$ & 1080 & 689 & 1019 \\
${ }^{17} \mathrm{O}^{16} \mathrm{O}^{17} \mathrm{O}$ & 1087 & 684 & 1031 \\
${ }^{17} \mathrm{O}^{17} \mathrm{O}^{18} \mathrm{O}$ & 1063 & 672 & 1006 \\
${ }^{17} \mathrm{O}^{18} \mathrm{O}^{17} \mathrm{O}$ & 1057 & 677 & 995 \\
${ }^{18} \mathrm{O}^{18} \mathrm{O}^{16} \mathrm{O}$ & 1057 & 677 & 996 \\
${ }^{18} \mathrm{O}^{16} \mathrm{O}^{18} \mathrm{O}$ & 1072 & 668 & 1019 \\
${ }^{18} \mathrm{O}^{18} \mathrm{O}^{17} \mathrm{O}$ & 1049 & 669 & 990 \\
${ }^{18} \mathrm{O}^{17} \mathrm{O}^{18} \mathrm{O}$ & 1056 & 665 & 1001 \\
${ }^{16} \mathrm{O}^{17} \mathrm{O}^{18} \mathrm{O}$ & 1072 & 681 & 1013 \\
${ }^{16} \mathrm{O}^{18} \mathrm{O}^{17} \mathrm{O}$ & 1065 & 685 & 1002 \\
${ }^{17} \mathrm{O}^{16} \mathrm{O}^{18} \mathrm{O}$ & 1080 & 676 & 1025 \\
\hline${ }^{18}$ & & & \\
\hline
\end{tabular}

\section{NUMERICAL RESULTS}

\section{A. Isotopic enrichments}

To calculate the various isotopic enrichments and the recombination rate constants the values for the moments of inertia and vibration frequencies of the various isotopomers are needed. The former are known. A formula for the latter is available $^{52}$ for symmetric isotopomers XYX and XXX. We have tested it using known values where available, ${ }^{33,53,54}$ and have also derived an approximate formula for asymmetric isotopomers, which we have also tested using known values. ${ }^{33,53,54}$ In each case the calculated and experimental results agree to within less than $4 \mathrm{~cm}^{-1}$, which is quite adequate for our calculation of $\rho$. The formula and tests will be submitted for publication elsewhere. The calculated isotopomeric vibrational frequencies used in the present calculation are given in Table I.

Calculated results for isotopic enrichments for "scrambled" systems with large concentrations of heavy isotopes are presented in Table II and Fig. 2 for $\eta=1.15$. They are obtained from individual isotopomeric rate constants and Eqs. (4.18a), (4.18b), and (4.26) of paper I. In order to make a comparison with the existing experimental data, we have used the ratios of rate constants calculated at low temperature, where the phase space theory transition state is valid, together with the equilibrium constants from $300 \mathrm{~K}$, where the experiments are performed. This approximation should be valid to the extent that ratios of the rate constants are not strongly temperature dependent, apart from any dependence of $\eta$ on $T$ (more precisely on energy). We use a value of $\eta$ chosen to fit the experimental data at $300 \mathrm{~K}$.

For the calculation of absolute rate constants the frequencies in Table I are needed, together with the deactivating collision frequencies, $\omega$. We have used a Lennard-Jones collision frequency with a unit collision efficiency. It is worth noting that in relative rate constants and in $\delta$ the ratio of collision frequencies is independent of both the temperature and the choice of Lennard-Jones parameters, and reduces to
TABLE II. Calculated and experimental isotopic enrichments at $300 \mathrm{~K}$ and room temperature.

\begin{tabular}{lcrrrr}
\hline \hline $\begin{array}{c}\text { Isotope } \\
\text { combination }\end{array}$ & Experiment $^{\mathrm{a}}$ & $\begin{array}{c}\text { Calc. }^{\mathrm{b}}(\%) \\
\eta=1.15\end{array}$ & $\begin{array}{c}\text { Calc. }^{\mathrm{b}}(\%) \\
\eta=1\end{array}$ & $\begin{array}{c}\text { Calc. }^{\mathrm{c}}(\%) \\
\eta=1.15\end{array}$ & $\begin{array}{c}\text { Calc. }^{\mathrm{c}}(\%) \\
\eta=1\end{array}$ \\
\hline${ }^{16} \mathrm{O}^{16} \mathrm{O}^{16} \mathrm{O}$ & 0.0 & 0.0 & 0.0 & 0.0 & 0.0 \\
${ }^{17} \mathrm{O}^{17} \mathrm{O}^{17} \mathrm{O}$ & -1.8 & -1.4 & -1.4 & -2.2 & -2.2 \\
${ }^{18} \mathrm{O}^{18} \mathrm{O}^{18} \mathrm{O}$ & -4.6 & -5.5 & -5.5 & -5.2 & -5.2 \\
${ }^{16} \mathrm{O}^{16} \mathrm{O}^{17} \mathrm{O}^{\mathrm{d}}$ & 11.3 & 12.7 & 2.4 & 10.4 & 0.4 \\
${ }^{16} \mathrm{O}^{16} \mathrm{O}^{18} \mathrm{O}^{\mathrm{d}}$ & 13.0 & 14.5 & 4.1 & 10.9 & 0.9 \\
${ }^{17} \mathrm{O}^{17} \mathrm{O}^{16} \mathrm{O}^{\mathrm{d}}$ & 12.1 & 12.0 & 1.7 & 9.7 & -0.4 \\
${ }^{17} \mathrm{O}^{17} \mathrm{O}^{18} \mathrm{O}^{\mathrm{d}}$ & 9.5 & 10.9 & 0.7 & 7.9 & -1.8 \\
${ }^{18} \mathrm{O}^{18} \mathrm{O}^{16} \mathrm{O}^{\mathrm{d}}$ & 14.4 & 13.1 & 2.4 & 9.4 & -0.8 \\
${ }^{18} \mathrm{O}^{18} \mathrm{O}^{17} \mathrm{O}^{\mathrm{d}}$ & 8.3 & 8.8 & -1.2 & 6.6 & -3.2 \\
${ }^{16} \mathrm{O}^{17} \mathrm{O}^{18} \mathrm{O}^{\mathrm{d}}$ & 18.1 & 18.9 & 3.4 & 15.0 & 0.0 \\
\hline
\end{tabular}

${ }^{a}$ Experimental data at $300 \mathrm{~K}$ are from Mauersberger et al., Ref. 19.

${ }^{\mathrm{b} C a l c u l a t e d}$ from Eqs. (4.18a), (4.18b), and (4.26) of paper I. The definition of enrichment is given by Eq. (4.13) in paper I. In order to make a comparison to the data at $300 \mathrm{~K}$, the rate constant ratios from $140 \mathrm{~K}$ were used together with the equilibrium constants at $300 \mathrm{~K}$. This assumption invokes the approximation that there is only a weak temperature dependence of the rate constant ratios.

${ }^{\mathrm{c}}$ As in footnote $\mathrm{b}$, but the calculated rate constants are those at $300 \mathrm{~K}$.

${ }^{\mathrm{d}}$ Enrichment is for all possible isotopomers.

$$
\frac{\omega^{\prime}}{\omega}=\left(\frac{\mu_{\mathrm{O}_{3}, M}}{\mu_{\mathrm{O}_{3}^{\prime}, M}}\right)^{1 / 2}
$$

when the $\mu_{\mathrm{O}_{3}, M}^{\prime}$ denotes the $\mu$ for some isotopomer of $\mathrm{O}_{3}, \mathrm{O}_{3}^{\prime}$.

\section{B. Individual rate constant ratios}

The results for the experimental individual rate constants for $\mathrm{X}+\mathrm{YY}$ relative to ${ }^{16} \mathrm{O}+{ }^{16} \mathrm{O}_{2}$ are plotted in Fig. 3 versus the ratios of rotational constants of the diatomic molecules in the two exit channels, prompted by the theoretical model in the present paper. The straight line there is merely to guide the eye. Because of correlation of differences in diatomic rotational constants with differences in zero-point energies and with ratios of reduced masses [all isotopomers obey the relation of $(a, b)$ and $\left(E_{0, a}, E_{0, b}\right)$ seen in Fig. 1] the plot in Fig. 3 could also have been vs the ratio of reduced masses in the two channels, or vs the difference in zero-point energies

\section{Isotopic Enrichments}

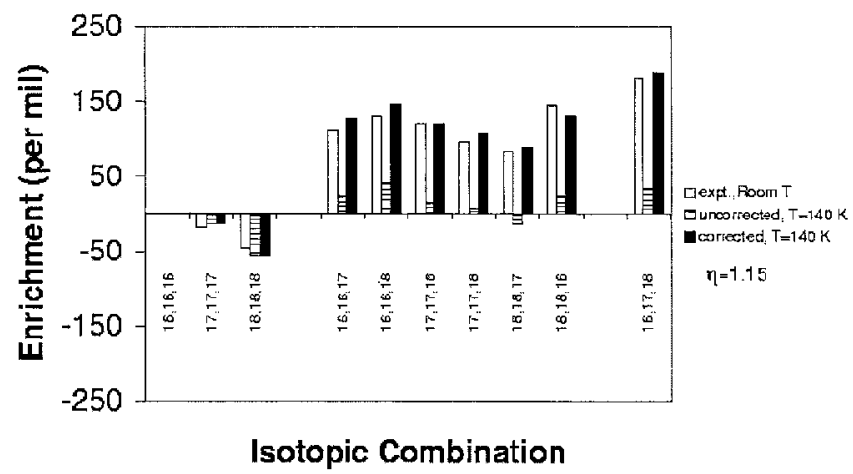

FIG. 2. Experimental and calculated (using rate constant ratios at $140 \mathrm{~K}$ ) isotopic enrichments for scrambled systems heavily enriched in heavy isotopes at room temperature, with $\eta=1.15$. 


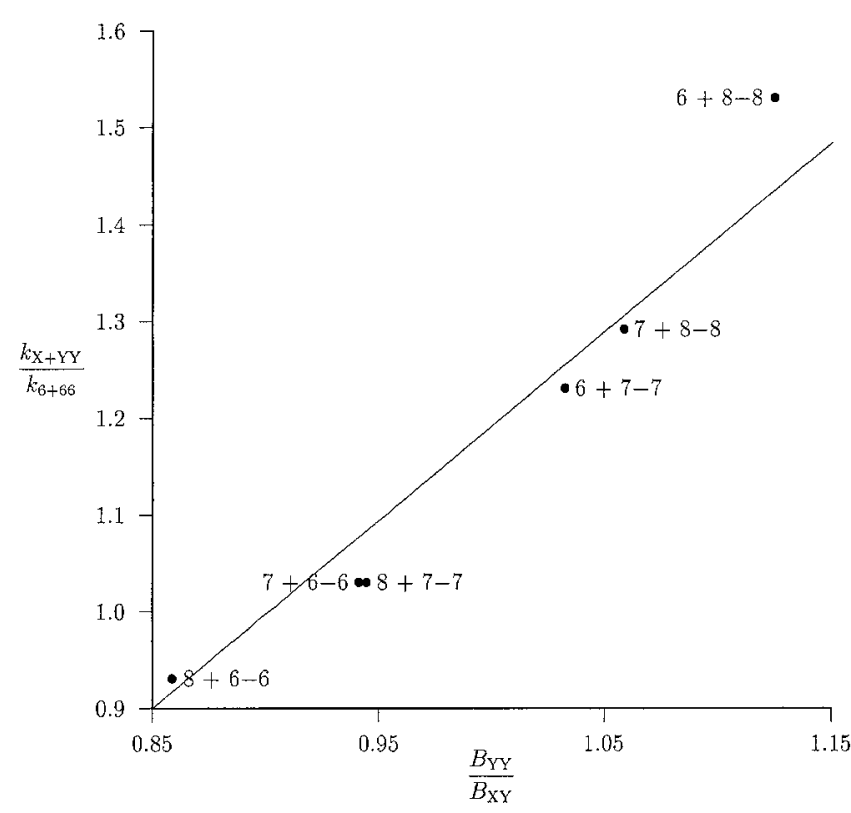

FIG. 3. Experimental rate constants for $\mathrm{X}+\mathrm{YY}$ reactions, relative to ${ }^{16} \mathrm{O}+{ }^{16} \mathrm{O}_{2}$ vs ratio of diatomic rotational constants, e.g., $B_{D, 88} / B_{D, 68}$ for the ${ }^{16} \mathrm{O}+{ }^{18} \mathrm{O}_{2}$ reaction. The straight line is a guide the eye. The plot could equally be made vs differences in zero-point energies or versus differences in reduced masses in the two exit channels, since all three quantities are related, as noted in Sec. VI.

of the diatomic species in the two channels, which can be shown to exhibit a parallel behavior. The results for the individual ratios of rate constants, $k_{\text {rec }}^{0}$ 's, obtained at $140 \mathrm{~K}$ from Eq. (2.1) are given in Figs. 4 and 5, as well as in Tables III and IV. In the tables the results for $\eta=1.15$ are compared with those at $\eta=1.0$. The results represent predictions for

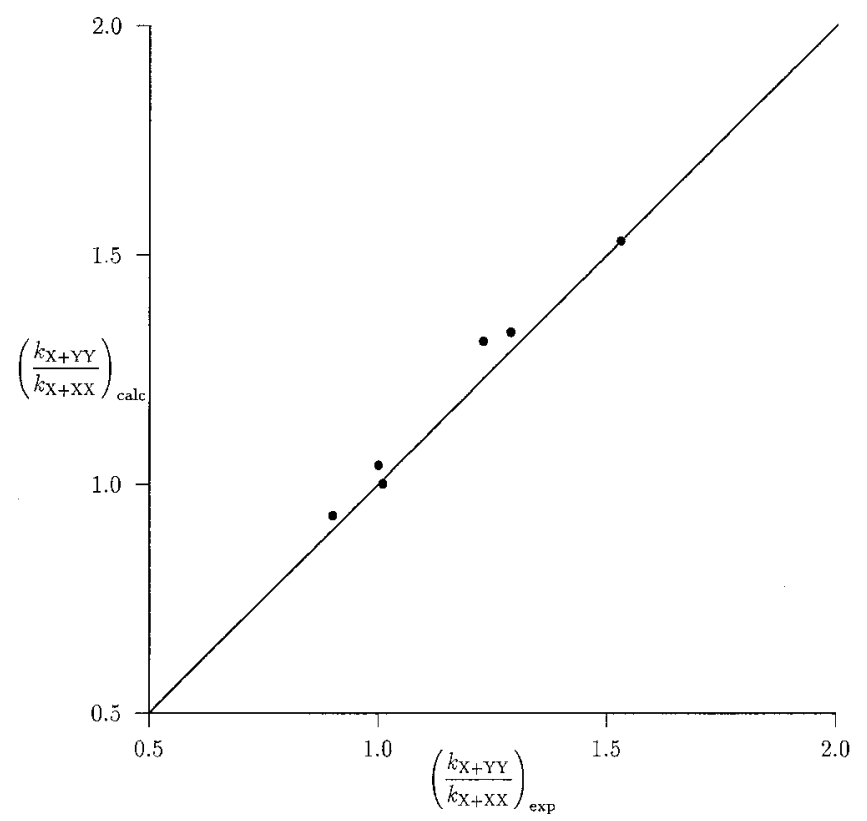

FIG. 4. Comparison of calculated and experimental relative atom+homonuclear diatomic rate ratios, $k_{\mathrm{X}+\mathrm{YY}} / k_{\mathrm{X}+\mathrm{XX}}$, with $\eta=1.15$. Experimental results are at room temperature, calculated results for these ratios of rate constants are at $140 \mathrm{~K}$. The line would represent perfect agreement of the ratios.

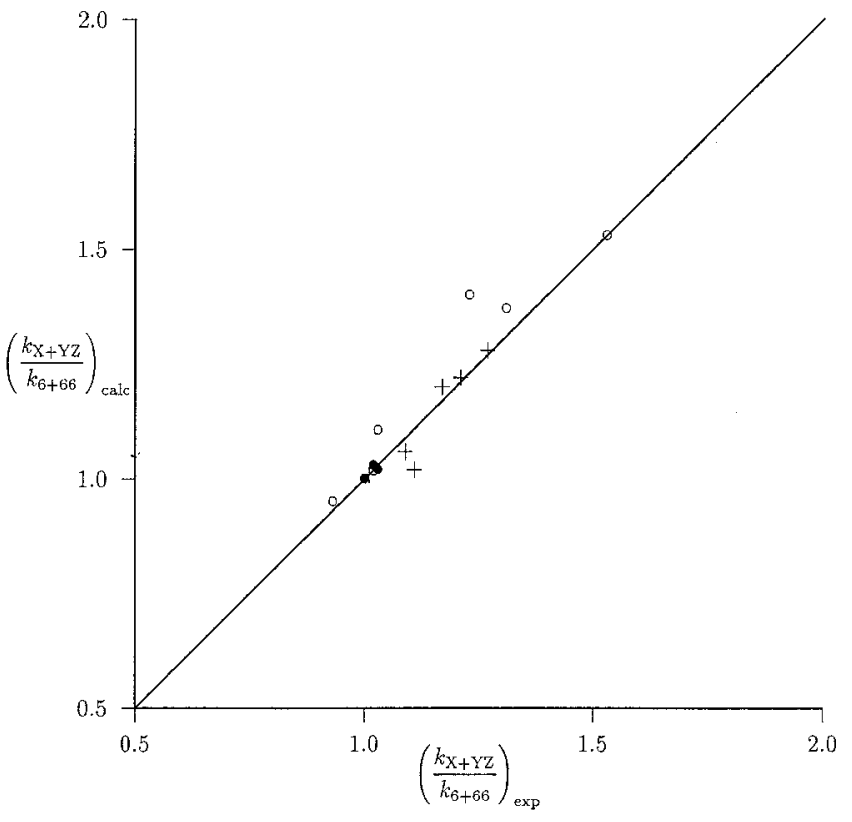

FIG. 5. Comparison of calculated and experimental relative atom + diatomic rate constant ratios, $k_{\mathrm{X}+\mathrm{YZ}} / k_{6+66}$ with $\eta=1.15$. $\bullet, \mathrm{X}+\mathrm{XX}$ reactions, $\mathrm{O}$, $\mathrm{X}+\mathrm{YY}$ reactions,,$+ \mathrm{X}+\mathrm{XY}$ reactions (sum of both channels). As in Fig. 4 the experimental results are at room temperature and the calculated results for these ratios of rate constants are at $140 \mathrm{~K}$. The line would represent perfect agreement of the ratios.

future data on these unscrambled systems at low temperatures, remembering that $\eta$ itself may be lifetime dependent and hence temperature dependent.

\section{Rate constants for symmetric and asymmetric channels}

Most recently, the rate constant ratios for the symmetric and asymmetric channels for the reactions involving ${ }^{16} \mathrm{O}^{18} \mathrm{O}$ have been measured. ${ }^{27}$ The experimental rate constant ratios (compared to ${ }^{16} \mathrm{O}+{ }^{16} \mathrm{O}^{16} \mathrm{O}$ ) for the symmetric and asymmetric channels of the ${ }^{16} \mathrm{O}+{ }^{16} \mathrm{O}^{18} \mathrm{O}$ reaction are 0.54 and 0.73 at room temperature, respectively. ${ }^{27,55}$ The rate constant ratios we obtain for the symmetric and asymmetric channels, albeit at somewhat lower temperatures where phase space theory is accurate, are 0.50 and 0.68 , using a factor of $\eta=1.15$ for the asymmetric channel. For the ${ }^{18} \mathrm{O}+{ }^{16} \mathrm{O}^{18} \mathrm{O}$ reaction the rate constant ratios for the symmetric and asymmetric channels are 0.52 and 0.46 , respectively, ${ }^{27,55}$ compared with calculated values of 0.51 and 0.52 . The complete set of specific rate

TABLE III. Relative rate coefficients of atom plus homonuclear diatomic formation channels $(\mathrm{X}+\mathrm{YY} \rightarrow \mathrm{XYY})$ at low pressure. Experimental results are at room temperature, calculated results are at $140 \mathrm{~K}$.

\begin{tabular}{cccc}
\hline \hline Reaction & Expt. & $\eta=1.15$ & $\eta=1$ \\
\hline${ }^{16} \mathrm{O}+{ }^{18} \mathrm{O}_{2} /{ }^{16} \mathrm{O}+{ }^{16} \mathrm{O}_{2}$ & $1.53 \pm 0.03$ & 1.53 & 1.33 \\
${ }^{17} \mathrm{O}+{ }^{18} \mathrm{O}_{2} /{ }^{17} \mathrm{O}+{ }^{17} \mathrm{O}_{2}$ & $1.29 \pm 0.07$ & 1.33 & 1.16 \\
${ }^{16} \mathrm{O}+{ }^{17} \mathrm{O}_{2} /{ }^{16} \mathrm{O}+{ }^{16} \mathrm{O}_{2}$ & $1.23 \pm 0.03$ & 1.31 & 1.14 \\
${ }^{17} \mathrm{O}+{ }^{16} \mathrm{O}_{2} /{ }^{17} \mathrm{O}+{ }^{17} \mathrm{O}_{2}$ & $1.01 \pm 0.05$ & 1.00 & 0.87 \\
${ }^{18} \mathrm{O}+{ }^{17} \mathrm{O}_{2} /{ }^{18} \mathrm{O}+{ }^{18} \mathrm{O}_{2}$ & $1.00 \pm 0.06$ & 1.04 & 0.90 \\
${ }^{18} \mathrm{O}+{ }^{16} \mathrm{O}_{2} /{ }^{18} \mathrm{O}+{ }^{18} \mathrm{O}_{2}$ & $0.90 \pm 0.03$ & 0.93 & 0.81 \\
\hline \hline
\end{tabular}

${ }^{\mathrm{a}}$ From Mauersberger et al., Ref. 26. 
TABLE IV. Reaction rate coefficients for ozone formation processes relative to ${ }^{16} \mathrm{O}+{ }^{16} \mathrm{O}_{2} \rightarrow{ }^{16} \mathrm{O}_{3}$ at low pressure.

\begin{tabular}{cccc}
\hline \hline Reaction & Expt. ${ }^{\mathrm{a}}$ & $\eta=1.15^{\mathrm{b}}$ & $\eta=1^{\mathrm{b}}$ \\
\hline${ }^{16} \mathrm{O}+{ }^{16} \mathrm{O}_{2}$ & 1.00 & 1.00 & 1.00 \\
${ }^{17} \mathrm{O}+{ }^{17} \mathrm{O}_{2}$ & 1.02 & 1.03 & 1.03 \\
${ }^{18} \mathrm{O}+{ }^{18} \mathrm{O}_{2}$ & 1.03 & 1.02 & 1.02 \\
${ }^{18} \mathrm{O}+{ }^{16} \mathrm{O}_{2}$ & 0.93 & 0.95 & 0.83 \\
${ }^{17} \mathrm{O}+{ }^{16} \mathrm{O}_{2}$ & 1.03 & 1.02 & 0.89 \\
${ }^{18} \mathrm{O}+{ }^{17} \mathrm{O}_{2}$ & 1.03 & 1.06 & 0.93 \\
${ }^{17} \mathrm{O}+{ }^{18} \mathrm{O}_{2}$ & 1.31 & 1.37 & 1.19 \\
${ }^{16} \mathrm{O}+{ }^{17} \mathrm{O}_{2}$ & 1.23 & 1.40 & 1.21 \\
${ }^{16} \mathrm{O}+{ }^{18} \mathrm{O}_{2}$ & 1.53 & 1.53 & 1.33 \\
${ }^{16} \mathrm{O}+{ }^{16} \mathrm{O}^{17} \mathrm{O}^{\mathrm{c}}$ & 1.17 & 1.20 & 1.11 \\
${ }^{16} \mathrm{O}+{ }^{16} \mathrm{O}^{18} \mathrm{O}^{\mathrm{c}}$ & 1.27 & 1.28 & 1.18 \\
${ }^{17} \mathrm{O}+{ }^{16} \mathrm{O}^{17} \mathrm{O}^{\mathrm{c}}$ & 1.11 & 1.02 & 0.96 \\
${ }^{17} \mathrm{O}+{ }^{17} \mathrm{O}^{18} \mathrm{O}^{\mathrm{c}}$ & 1.21 & 1.22 & 1.13 \\
${ }^{18} \mathrm{O}+{ }^{16} \mathrm{O}^{18} \mathrm{O}^{\mathrm{c}}$ & 1.01 & 1.01 & 0.95 \\
${ }^{18} \mathrm{O}+{ }^{17} \mathrm{O}^{18} \mathrm{O}^{\mathrm{c}}$ & 1.09 & 1.06 & 0.99 \\
${ }^{16} \mathrm{O}+{ }^{17} \mathrm{O}^{18} \mathrm{O}^{\mathrm{c}}$ & & 1.47 & 1.28 \\
${ }^{17} \mathrm{O}+{ }^{16} \mathrm{O}^{18} \mathrm{O}^{\mathrm{c}}$ & & 1.22 & 1.06 \\
${ }^{18} \mathrm{O}+{ }^{16} \mathrm{O}^{17} \mathrm{O}^{\mathrm{c}}$ & & 1.01 & 0.88 \\
\hline \hline
\end{tabular}

${ }^{a}$ From Mauersberger et al. Ref. 26, at room temperature.

${ }^{\mathrm{b}}$ Results calculated at $140 \mathrm{~K}$.

${ }^{\text {c}}$ The rate constant which appears here is the sum of both channels, e.g., both $\mathrm{X}+\mathrm{YZ} \rightarrow \mathrm{XYZ}$ and $\mathrm{X}+\mathrm{YZ} \rightarrow \mathrm{XZY}$. Each of the rate constants were determined separately, with the non-RRKM correction only applied to the symmetric channel, where applicable.

constant ratios is provided in Table V. The equilibrium constants used in obtaining the results in Table II and Fig. 2 are given in Table VI.

\section{Recombination rate constants for ${ }^{16} \mathrm{O}+{ }^{16} \mathrm{O}_{2} \rightarrow{ }^{16} \mathrm{O}_{3}$}

Calculated values for $k_{\text {rec }}^{0}$ and $k_{\text {rec }}^{\infty}$ for ${ }^{16} \mathrm{O}+{ }^{16} \mathrm{O}_{2} \rightarrow{ }^{16} \mathrm{O}_{3}$ are given in Table VII, and compared there with experimen-

TABLE V. Reaction rate coefficients for asymmetric and symmetric channels of recombination reactions, relative to ${ }^{16} \mathrm{O}+{ }^{16} \mathrm{O}_{2} \rightarrow{ }^{16} \mathrm{O}_{3}$ at low pressure.

\begin{tabular}{|c|c|c|c|}
\hline Reaction & Expt. $^{\mathrm{a}}$ & $\eta=1.15^{\mathrm{b}}$ & $\eta=1^{\mathrm{b}}$ \\
\hline \multicolumn{4}{|l|}{ Symmetric products } \\
\hline${ }^{16} \mathrm{O}+{ }^{17} \mathrm{O}^{16} \mathrm{O} \rightarrow{ }^{16} \mathrm{O}^{17} \mathrm{O}^{16} \mathrm{O}$ & & 0.51 & 0.51 \\
\hline${ }^{16} \mathrm{O}+{ }^{18} \mathrm{O}^{16} \mathrm{O} \rightarrow{ }^{16} \mathrm{O}^{18} \mathrm{O}^{16} \mathrm{O}$ & $0.54 \pm 0.01$ & 0.52 & 0.52 \\
\hline${ }^{17} \mathrm{O}+{ }^{16} \mathrm{O}^{17} \mathrm{O} \rightarrow{ }^{17} \mathrm{O}^{16} \mathrm{O}^{17} \mathrm{O}$ & & 0.50 & 0.50 \\
\hline${ }^{17} \mathrm{O}+{ }^{18} \mathrm{O}^{17} \mathrm{O} \rightarrow{ }^{17} \mathrm{O}^{18} \mathrm{O}^{17} \mathrm{O}$ & & 0.52 & 0.52 \\
\hline${ }^{18} \mathrm{O}+{ }^{16} \mathrm{O}^{18} \mathrm{O} \rightarrow{ }^{18} \mathrm{O}^{16} \mathrm{O}^{18} \mathrm{O}$ & $0.52 \pm 0.01$ & 0.51 & 0.51 \\
\hline${ }^{18} \mathrm{O}+{ }^{17} \mathrm{O}{ }^{18} \mathrm{O} \rightarrow{ }^{18} \mathrm{O}^{17} \mathrm{O}^{18} \mathrm{O}$ & & 0.52 & 0.52 \\
\hline \multicolumn{4}{|l|}{ Asymmetric products ${ }^{\mathrm{c}}$} \\
\hline${ }^{18} \mathrm{O}+{ }^{17} \mathrm{O}^{16} \mathrm{O} \rightarrow{ }^{18} \mathrm{O}^{17} \mathrm{O}^{16} \mathrm{O}$ & & 0.49 & 0.42 \\
\hline${ }^{18} \mathrm{O}+{ }^{18} \mathrm{O}^{16} \mathrm{O} \rightarrow{ }^{18} \mathrm{O}^{18} \mathrm{O}^{16} \mathrm{O}$ & $0.46 \pm 0.03$ & 0.49 & 0.43 \\
\hline${ }^{17} \mathrm{O}+{ }^{18} \mathrm{O}^{16} \mathrm{O} \rightarrow{ }^{17} \mathrm{O}^{18} \mathrm{O}^{16} \mathrm{O}$ & & 0.53 & 0.46 \\
\hline${ }^{17} \mathrm{O}+{ }^{17} \mathrm{O}^{16} \mathrm{O} \rightarrow{ }^{17} \mathrm{O}^{17} \mathrm{O}^{16} \mathrm{O}$ & & 0.52 & 0.45 \\
\hline${ }^{18} \mathrm{O}+{ }^{18} \mathrm{O}^{17} \mathrm{O} \rightarrow{ }^{18} \mathrm{O}^{18} \mathrm{O}^{17} \mathrm{O}$ & & 0.54 & 0.47 \\
\hline${ }^{18} \mathrm{O}+{ }^{16} \mathrm{O}{ }^{17} \mathrm{O} \rightarrow{ }^{18} \mathrm{O}^{16} \mathrm{O}^{17} \mathrm{O}$ & & 0.52 & 0.45 \\
\hline${ }^{17} \mathrm{O}+{ }^{16} \mathrm{O}{ }^{18} \mathrm{O} \rightarrow{ }^{17} \mathrm{O}^{16} \mathrm{O}^{18} \mathrm{O}$ & & 0.69 & 0.60 \\
\hline${ }^{16} \mathrm{O}+{ }^{16} \mathrm{O}^{17} \mathrm{O} \rightarrow{ }^{16} \mathrm{O}^{16} \mathrm{O}^{17} \mathrm{O}$ & & 0.69 & 0.60 \\
\hline${ }^{17} \mathrm{O}+{ }^{17} \mathrm{O}^{18} \mathrm{O} \rightarrow{ }^{17} \mathrm{O}^{17} \mathrm{O}^{18} \mathrm{O}$ & & 0.70 & 0.61 \\
\hline${ }^{16} \mathrm{O}+{ }^{18} \mathrm{O}^{17} \mathrm{O} \rightarrow{ }^{16} \mathrm{O}^{18} \mathrm{O}^{17} \mathrm{O}$ & & 0.70 & 0.61 \\
\hline${ }^{16} \mathrm{O}+{ }^{16} \mathrm{O}^{18} \mathrm{O} \rightarrow{ }^{16} \mathrm{O}^{16} \mathrm{O}^{18} \mathrm{O}$ & $0.73 \pm 0.02$ & 0.76 & 0.66 \\
\hline${ }^{16} \mathrm{O}+{ }^{17} \mathrm{O}^{18} \mathrm{O} \rightarrow{ }^{16} \mathrm{O}^{17} \mathrm{O}^{18} \mathrm{O}$ & & 0.77 & 0.67 \\
\hline
\end{tabular}

a From Janssen et al., at room temperature, Ref. 27. See also Ref. 55 for a definition of the value of $k$ used.

${ }^{\mathrm{b}}$ Calculated at $140 \mathrm{~K}$.

${ }^{c}$ Reactions for asymmetric products are ordered in sequence of increasing zero-point energy difference.
TABLE VI. Calculated equilibrium constants for isotopic exchange reactions at $300 \mathrm{~K}$.

\begin{tabular}{ccc}
\hline \hline Reaction & $K_{\text {eq }}$ & Value \\
\hline${ }^{16} \mathrm{O}+{ }^{17} \mathrm{O}_{2} \rightleftharpoons{ }^{17} \mathrm{O}+{ }^{16} \mathrm{O}^{17} \mathrm{O}$ & $K_{6,77}^{7,67}$ & 1.92 \\
${ }^{16} \mathrm{O}+{ }^{18} \mathrm{O}_{2} \rightleftharpoons{ }^{18} \mathrm{O}+{ }^{16} \mathrm{O}^{18} \mathrm{O}$ & $K_{6,88}^{8,68}$ & 1.85 \\
${ }^{17} \mathrm{O}+{ }^{16} \mathrm{O}_{2} \rightleftharpoons{ }^{16} \mathrm{O}+{ }^{16} \mathrm{O}^{17} \mathrm{O}$ & $K_{7,66}^{6,67}$ & 2.08 \\
${ }^{17} \mathrm{O}+{ }^{18} \mathrm{O}_{2} \rightleftharpoons{ }^{18} \mathrm{O}+{ }^{17} \mathrm{O} \mathrm{O}^{18} \mathrm{O}$ & $K_{7,78}^{8,78}$ & 1.93 \\
${ }^{18} \mathrm{O}+{ }^{16} \mathrm{O}_{2} \rightleftharpoons{ }^{16} \mathrm{O}+{ }^{18} \mathrm{O}^{16} \mathrm{O}$ & $K_{8,68}^{6,68}$ & 2.16 \\
${ }^{18} \mathrm{O}+{ }^{17} \mathrm{O}_{2} \rightleftharpoons{ }^{17} \mathrm{O}+{ }^{17} \mathrm{O}^{18} \mathrm{O}$ & $K_{8,77}^{7,78}$ & 2.08 \\
${ }^{16} \mathrm{O}+{ }^{17} \mathrm{O} \mathrm{O}^{18} \mathrm{O} \rightleftharpoons{ }^{17} \mathrm{O}+{ }^{16} \mathrm{O}^{18} \mathrm{O}$ & $K_{6,78}^{7,68}$ & 0.96 \\
${ }^{16} \mathrm{O}+{ }^{17} \mathrm{O}^{18} \mathrm{O} \rightleftharpoons{ }^{18} \mathrm{O}+{ }^{16} \mathrm{O}^{17} \mathrm{O}$ & $K_{6,78}^{8,67}$ & 0.92 \\
${ }^{17} \mathrm{O}+{ }^{16} \mathrm{O}^{18} \mathrm{O} \rightleftharpoons{ }^{18} \mathrm{O}+{ }^{16} \mathrm{O}^{17} \mathrm{O}$ & $K_{7,68}^{8,67}$ & 0.96 \\
\hline \hline
\end{tabular}

tal values. The observed pressure dependence of $k_{\text {rec }}$ at room and nearby temperatures is complex, ${ }^{32}$ but the data at $130 \mathrm{~K}$ are simpler. Calculated results of $k_{\mathrm{rec}}^{0}$ and $k_{\mathrm{rec}}^{\infty}$ obtained using Eqs. (2.1) and (2.2) are given in the table. One uncertainty in the calculation of the absolute value of $k_{\mathrm{rec}}^{0}$ and its temperature dependence is the value and temperature dependence of $\omega$ and the efficiency of the deactivating collisions.

\section{E. Exchange rate constants}

The results from the application of Eq. (2.3) for the exchange rate constant at low temperature are given in Table VII. As Eq. (2.3) contains no explicit pressure dependence, these results are independent of such quantities as the collision rate and the collision efficiency, and can be independently compared to the existing experimental data on the exchange rate. In the present case, the calculated exchange rates are in reasonably close agreement with the experimentally observed ones at low temperature, indicating that the transition state is well represented by a phase space theory transition state. The disagreement at higher temperatures is indicative that the transition state may tighten somewhat at higher energies.

\section{F. Calculation of $\boldsymbol{k}_{\mathrm{rec}}^{\infty}$ from $\boldsymbol{k}_{\mathrm{ex}}$}

It is useful to use the very approximate estimate given by Eq. (2.6) and the experimental value of $k_{\text {ex }}$ to obtain a rough

TABLE VII. Calculated and experimental rate constants.

\begin{tabular}{ccccc}
\hline \hline$k$ & Reaction & $T(\mathrm{~K})$ & Expt. & Calc. \\
\hline$k_{\text {rec }}^{0}$ & ${ }^{16} \mathrm{O}_{2}+{ }^{16} \mathrm{O}+\mathrm{N}_{2} \rightarrow{ }^{16} \mathrm{O}_{3}+\mathrm{N}_{2}$ & 130 & $4^{\mathrm{c}}$ & $7.0^{\mathrm{b}}$ \\
$k_{\text {ex }}{ }^{\mathrm{d}}$ & ${ }^{16} \mathrm{O}+{ }^{18} \mathrm{O}_{2} \rightarrow{ }^{16} \mathrm{O}^{18} \mathrm{O}+{ }^{18} \mathrm{O}$ & 300 & $0.5^{\mathrm{c}}$ & $3.7^{\mathrm{b}}$ \\
$k_{\text {rec }}^{\infty}$ & & 300 & $5.6^{\mathrm{e}}$ & $8.1^{\mathrm{f}}$ \\
& ${ }^{16} \mathrm{O}+{ }^{16} \mathrm{O}_{2} \rightarrow{ }^{16} \mathrm{O}_{3}$ & 130 & $2.9^{\mathrm{e}}$ & $8.3^{\mathrm{f}}$ \\
& & 300 & $>8^{\mathrm{c}}$ & $19^{\mathrm{g}, \mathrm{h}}$ \\
\hline \hline
\end{tabular}

${ }^{a}$ Units are $10^{-33} \mathrm{~cm}^{6} \mathrm{~s}^{-1}$.

${ }^{\mathrm{b}}$ Calculated from Eq. (2.1).

${ }^{c}$ Experimental data from Hippler et al., Ref. 32.

${ }^{\mathrm{d}}$ Units are $10^{-12} \mathrm{~cm}^{3}$ molecule ${ }^{-1} \mathrm{~s}^{-1}$.

${ }^{\text {e}}$ Experimental data from Wiegell et al., Ref. 34.

${ }^{\mathrm{f}}$ Calculated from Eq. (2.3).

${ }^{g}$ Calculated from Eq. (2.2).

${ }^{\text {h}}$ Estimated to be 11 from the very approximate Eq. (2.6).

${ }^{\mathrm{i}}$ Estimated to be 5.8 from the very approximate Eq. (2.6). 
estimate of $k_{\text {rec }}^{\infty}$ and compare it with the observed values for ${ }^{16} \mathrm{O}+{ }^{16} \mathrm{O}_{2} \rightarrow{ }^{16} \mathrm{O}_{3}$. Only the data at $130 \mathrm{~K}$ appear to be uncomplicated. In applying Eq. (2.6), the isotopic exchange reaction used for the $k_{\mathrm{ex}}$ 's was ${ }^{16} \mathrm{O}+{ }^{18} \mathrm{O}_{2} \rightarrow{ }^{18} \mathrm{O}+{ }^{16} \mathrm{O}^{18} \mathrm{O}$. Experimental and calculated values of $k_{\text {rec }}^{\infty}$ are given in Table VII. The experimental $k_{\mathrm{ex}}$ at $130 \mathrm{~K}$, using the formula given by Wiegell et al., ${ }^{34}$ is $5.6 \times 10^{-12} \mathrm{~cm}^{3}$ molecule $\mathrm{s}^{-1}$. The value of $k_{\text {rec }}^{\infty}$ predicted from Eq. (2.6) is then 11 $\times 10^{-12} \mathrm{~cm}^{3}$ molecule $\mathrm{s}^{-1} \mathrm{~s}^{-1}$ while the observed value at 130 $\mathrm{K}$ is $18 \times 10^{-12} \mathrm{~cm}^{3}$ molecule $\mathrm{s}^{-1}$.

At $300 \mathrm{~K}$, the thermal data of Hippler et al. ${ }^{32}$ show complexities not typical in unimolecular falloff behavior. The results appear to approach a limit, and then have an upsweep to a higher value with increased pressure. The value of $k_{\text {rec }}^{\infty}$ predicted from Eq. (2.6) at $300 \mathrm{~K}$ is 5.8 $\times 10^{-12} \mathrm{~cm}^{3}$ molecule $\mathrm{s}^{-1}$, but the upsweep indicates that a value higher than 4 will be reached.

\section{DISCUSSION}

We note that the recent experiments on the rate constants of the individual channels ${ }^{27}$ (symmetric vs asymmetric products) validate the present assumption that the addition is primarily "end on," with very little contribution, if any, to the products resulting from insertion of an oxygen atom into the $\mathrm{O}_{2}$ bond. (The current experimental results indicate that insertion into the bond is less than $<1 \%$ of the end-on attack.)

As noted previously, the PST transition state utilized in these calculations is quite loose, and cannot correctly account for the well known ${ }^{56-68}$ tightening of the transition state which typically occurs at higher energies, and thus at higher temperatures. At low pressure the recombination rate depends weakly on the transition state (via the effective energy barriers in the exit and entrance channels) and also on the density of states, $\rho$ of the ozone molecule and on the deactivation collision efficiency. To assess better the validity of the PST transition state it is best to examine the numerical results which are independent of pressure and depend only on the transition state, namely the exchange rates and the high pressure rate constants, Eqs. (2.2) and (2.3).

The data for the high pressure rate constants at high temperature appears to be atypical in its falloff behavior for bimolecular recombination reactions, and thus, such comparisons may be suspect. Nevertheless, we include them here as a matter of completeness. At $130 \mathrm{~K}$ the high pressure recombination rate for the $\mathrm{O}+\mathrm{O}_{2} \rightarrow \mathrm{O}_{3}$ reaction approaches a limit of approximately ${ }^{32} 1.9 \times 10^{-11} \mathrm{~cm}^{3} \mathrm{~s}^{-1}$. This compares favorably with the numerically calculated value of 1.9 $\times 10^{-11} \mathrm{~cm}^{3} \mathrm{~s}^{-1}$. The corresponding values at room temperature $\operatorname{are}^{32} 3.9-4.6 \times 10^{-12} \mathrm{~cm}^{3} \mathrm{~s}^{-1}$, depending on the bath gas collision partner used, and $1.8 \times 10^{-11} \mathrm{~cm}^{3} \mathrm{~s}^{-1}$, indicating that the transition state is somewhat tighter at higher temperatures, perhaps by a factor of as much as 5 . However, the room temperature recombination rate data are, as noted earlier, complex.

The experiments for the exchange rates do not contain this complication and thus we can now make a ready comparison between the experimental and the calculated results. At $140 \mathrm{~K}$, the experimental value of the exchange rate for
${ }^{16} \mathrm{O}+{ }^{18} \mathrm{O}_{2}$ is about ${ }^{34} 5.2 \times 10^{-12} \mathrm{~cm}^{3} \mathrm{~s}^{-1}$, compared with the value calculated from Eq. (2.3), $8.1 \times 10^{-12} \mathrm{~cm}^{3} \mathrm{~s}^{-1}$. At 300 $\mathrm{K}$, the calculated value is $8.3 \times 10^{-12} \mathrm{~cm}^{3} \mathrm{~s}^{-1}$, where the experimental result is about $2.9 \times 10^{-12} \mathrm{~cm}^{3} \mathrm{~s}^{-1}$, indicating that the transition state may be tighter by perhaps a factor of 3-4 at the higher temperature. Nonetheless, the results at low temperature show that PST is a good approximation for low energies.

The results of the model for phase space partitioning, together with the non-RRKM correction for the symmetric molecules, yield several interesting results. In particular the general features of the "mass-independent" enrichments for the scrambled systems are well reproduced by the calculation, as seen in Fig. 2. As noted previously, these results are based on the assumption that the rate constant ratios calculated at low temperature, where PST appears to be valid, are the same as those obtained at room temperature, where the transition state may be somewhat tighter and PST does not provide its best representation. In essence, this assumption means that the calculated rate constant ratios are assumed to have a small temperature dependence over this temperature region, apart from any dependence of $\eta$ on temperature.

The highest enrichment, both in the experiment and in the calculations, occurs for the isotopomers which contain all three isotopes, ${ }^{16} \mathrm{O},{ }^{17} \mathrm{O}$, and ${ }^{18} \mathrm{O}$. The only parameter used for the figure was $\eta=1.15$, which was chosen to have the same value as that in Tables III and IV and Figs. 4 and 5 for the unscrambled systems. The importance of $\eta$ and the relative unimportance of differences in zero-point energies (and diatomic rotational constants and centrifugal potentials) in their effect on the enrichments, $E_{i j j}$, is seen by examining the striped blocks in Fig. 2: In each case the calculated enrichment is small when $\eta=1$. That is, the effect of differences in zero-point energies, rotational densities of states and in centrifugal potentials in the two exit channels is "washed out" for $E_{i j j}$ (and for ${ }^{q} \delta$ ), since the partitioning effect has disappeared: the "partitioning factor" for the scrambled system is now unity in Eqs. (2.12) and (2.13) for $k_{\mathrm{rec}}^{\mathrm{eff}, a}$ and $k_{\mathrm{rec}}^{\mathrm{eff}, b}$, which appear in the Eq. (2.20) for $E_{i j j}$.

The negative enrichment of the ${ }^{17} \mathrm{O}^{17} \mathrm{O}^{17} \mathrm{O}$ and ${ }^{18} \mathrm{O}^{18} \mathrm{O}^{18} \mathrm{O}$ molecules are also well reproduced in Fig. 2. These negative values of $E_{j j j}$ in Fig. 2 for $j=7$ and 8 are not due to a decrease in $k_{\text {rec }}^{0}$ for ${ }^{17} \mathrm{O}+{ }^{17} \mathrm{O}_{2}$ or ${ }^{18} \mathrm{O}+{ }^{18} \mathrm{O}_{2}$. Indeed, these $k_{\text {rec }}^{0}$ 's are seen in Table IV to be slightly larger than the $k_{\text {rec }}^{0}$ for ${ }^{16} \mathrm{O}+{ }^{16} \mathrm{O}_{2}$. Instead, the negative enrichments $E_{j j j}$ for $j=7$ and 8 can be shown from Eq. (2.21) for $E_{j j j}$ to be due to a reduced concentration of ${ }^{17} \mathrm{O}_{2}$ and ${ }^{18} \mathrm{O}_{2}$ in the scrambled mixture, as reflected in the presence of the equilibrium constant $K_{6, j j}^{j, 67}$ appearing in the first half of that equation. Similarly, the appreciably negative values for $\eta=1$ in Fig. 2 for $E_{i j j}$ for $i=7, j=8$ and for $i=8, j=7$ are due to the same $K_{6, j j}^{j, 6 j}$ appearing in Eq. (2.20).

The comparison of experimental and calculated results for the recombination rates for the unscrambled systems are given for $\mathrm{X}+\mathrm{YY} \rightarrow \mathrm{XYY}$ in Table III and Fig. 4, and the results for all recombination reactions are given in Table IV and Fig. 5. We see that these results show very large massdependent isotope effects of up to approximately $50 \%$, in contrast with the nature of the enrichment observed in the 
scrambled systems (where the enrichments are typically less than about $15 \%$ and approximately mass independent). Similar remarks apply to the more general reactions $\mathrm{X}+\mathrm{YZ} \rightarrow \mathrm{XYZ}+\mathrm{XZY}$ in Table IV and Fig. 5. In physical terms, these large isotope effects for unscrambled systems in the present model have their origin in the partitioning factors. By comparison with the results for $\eta=1$, it is seen that for these individual rate constants the partitioning factor effect contributes about $50 \%$ to the differences in $k_{\text {rec }}^{0}$ 's while the $\eta \neq 1$ ( 1.15 in these tables) contributes the other $50 \%$.

Upon examining Tables III and IV more closely we see that the various results in these tables can be understood in terms of Fig. 1. In Fig. 1 we make an estimate that $J \sim \ell$, which is used only for the purposes of this illustration and not in the actual calculations. When the diatomic reactant has a lower zero-point energy than the diatomic molecule in the other (exit) channel, the integration is over the regions I and III in Fig. 1 where in I the partitioning factor $Y_{a}^{\dagger}$ is unity, while in III it is $N_{a}^{\dagger} /\left(N_{a}^{\dagger}+N_{b}^{\dagger}\right)$. Thus, at low and accessible E's and $\ell^{3}$, s there is a significant contribution from the $Y_{a}^{\dagger}$ $=1$ region, causing $k_{\text {rec }}^{0, a}$ to be high. Moreover, the diatomic reactant with a lower zero-point energy also has a higher moment of inertia and so a higher density of rotational states and a higher partitioning factor. Indeed, all three factors, the ratio of the diatomic rotational constants for $\mathrm{X}+\mathrm{YZ}$ to that for $X Y+Z$, the ratio of the reduced mass of $X+Y Z$ to that of $\mathrm{XY}+\mathrm{Z}$, and the ratio of zero-point energies of $\mathrm{XY}$ to $\mathrm{YZ}$, are all closely related: The first two equal $m_{\mathrm{X}}\left(m_{\mathrm{Y}}\right.$ $\left.+m_{\mathrm{Z}}\right) / m_{\mathrm{Z}}\left(m_{\mathrm{X}}+m_{\mathrm{Y}}\right)$, and the third equals the square root of this quantity. Accordingly, all of these differences in properties of the two channels are related to each other. Effectively, at low energies $E$ and at low $\ell^{3}$, s i.e., when region $I$ is important, the second exit channel is energetically excluded and so the excited molecule has a longer lifetime. It has a greater chance to be deactivated, therefore, by collision and so has a $k_{\text {rec }}^{0, a}$ ratio higher than unity (in the $\eta=1$ calculations).

In contrast, when the diatomic reactant has a higher zero-point energy than the diatomic molecule in the other (exit) channel, the integration is over the regions II and III in Fig. 1, where in II the partitioning factor $Y_{b}^{\dagger}$ is unity, while in III it is $N_{b}^{\dagger} /\left(N_{a}^{\dagger}+N_{b}^{\dagger}\right)$. The region where the relevant $Y_{b}^{\dagger}$ $=1$ (region II) is now both in terms of $E$ and $\ell^{3}$ less accessible, and so now the $k_{\text {rec }}^{0, b}$ ratio is expected to be less than unity. These ratios are, in Tables III and IV, all relative to some symmetric recombination reaction (all three atoms consisting of a single isotope). For this latter reaction, the two lines coalesce in Fig. 1 into a single line, since $E_{0, a}=E_{0, b}$ and the slope $a=b$. The exclusive regions I and II have now disappeared, and in III there is, in effect, a partitioning factor of $1 / 2$, corresponding to a symmetry number. Thus, the exclusive regions have disappeared and with it have the significant deviations (for $\eta=1$ ) of the ratios from unity. The role of $\eta \neq 1$ is superimposed on the trends in these two cases.

We note that the calculated results for the ratios of the rate constants are approximately temperature independent when calculated with the loose transition state of the present model. Such approximate temperature independence of the rate constant ratios is evident in the results of a similar cal-
Isotopic Enrichments

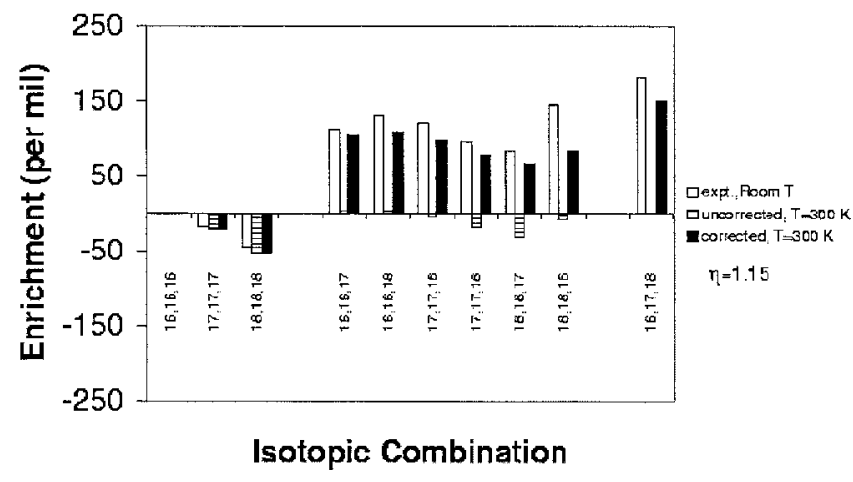

FIG. 6. Experimental and calculated (using rate constant ratios at $300 \mathrm{~K}$ ) isotopic enrichments for scrambled systems heavily enriched in heavy isotopes at room temperature, with $\eta=1.15$. No attempt was made to readjust $\eta$ from the value in Fig. 2 to obtain a better fit for this plot.

culation at room temperature, in Fig. 6. In this case it is known, by a direct comparison between the calculated and experimental results, that the transition state is too loose by a factor of 3-4, yet similar trends are observed in the calculated and experimental results.

A key factor in Fig. 2 is seen to be $\eta$, since the striped results $(\eta=1)$ show very small enrichments $E$, in contrast with the black results $(\eta=1.15)$ and also with experiment. The origin of $\eta$ is attributed in this paper to a non-RRKM effect, but the calculated results in these tables and figures are, as noted earlier, independent of the exact origin of $\eta$. The non-RRKM effect we have supposed arises from fewer resonance couplings in symmetric molecules where some portion of the ozone phase space in such molecules is assumed to be only weakly connected or disconnected from the phase space leading to the exit channels. Thereby, the symmetric molecule has a lower effective $\rho$ in Eq. (2.1) and hence a lower $k_{\text {rec }}^{0}$. For comparison we have given in Fig. 7 the enrichment plot for $\eta=1.30$ for comparison with Fig. 2 where $\eta=1.15$.

In summary, we believe that the data for individual recombination rate constants in Tables III and IV and Figs. 4

\section{Isotopic Enrichments}

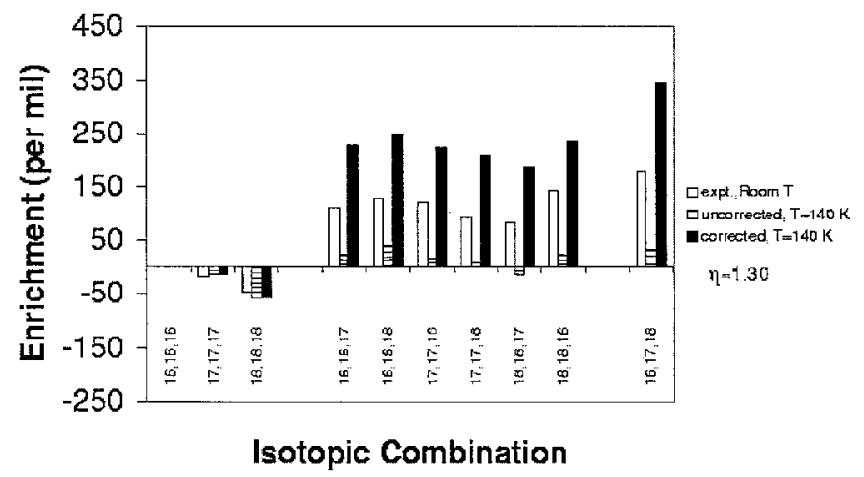

FIG. 7. Experimental and calculated (using rate constant ratios at $140 \mathrm{~K}$ ) isotopic enrichments for scrambled systems heavily enriched in heavy isotopes at room temperature, with $\eta=1.30$. 
and 5 are consistent with the above concepts, as are the "mass-independent" enrichment results for scrambled systems given in Fig. 2. The agreement with experiment is by no means perfect, but considering the various approximations made, we regard it as encouraging.

It is known that loose transition state theory, or phase space theory, does not agree with the negative temperature dependence so often seen in bimolecular association reactions. ${ }^{56-68}$ Phase space theory, for example, gives Eq. (3.6) with its positive $T^{1 / 6}$ dependence (excluding the temperature dependence of the electronic partition function), instead of the stronger negative temperature dependence found, for example, in the exchange reactions at low pressure [which correspond to the high pressure recombination rates, e.g., as in Eq. (2.6)]. The shortcoming of PST or loose transition state theory is that in the actual system the transition state moves inward with increasing energy and so with increasing temperature, resulting in a reduced rate. ${ }^{5-68}$ The hindered rotations in the transition state, evolving from the free rotations of the diatomic molecule, play a key role. We plan to explore this application of variational RRKM theory in an extension of the present study. We have not discussed pressure $^{12,13}$ effects, but plan to do so in a later publication.

\section{ACKNOWLEDGMENTS}

It is a pleasure to acknowledge the support of this research by the National Science Foundation and the award of a James W. Glanville Postdoctoral Fellowship in Chemistry at Caltech to one of us (B.C.H.). We have enjoyed helpful discussions with Professor Konrad Mauersberger and Professor Mark Thiemens and with Dr. Jürgen Günther. It is a pleasure to dedicate this paper to our colleague Professor Jürgen Troe, on the occasion of his sixtieth birthday. This paper was presented in part at the International Conference of Stable Isotopes, Carry le Rouet, France, June 20-25, 1999.

${ }^{1}$ B. C. Hathorn and R. A. Marcus, J. Chem. Phys. 111, 4087 (1999), denoted as paper I.

${ }^{2}$ R. N. Clayton, L. Grossman, and T. K. Mayeda, Science 182, 485 (1973).

${ }^{3}$ J. E. Heidenreich III and M. H. Thiemens, J. Chem. Phys. 78, 892 (1983).

${ }^{4}$ J. E. Heidenreich III and M. H. Thiemens, J. Chem. Phys. 84, 2129 (1986).

${ }^{5}$ K. Mauersberger, Geophys. Res. Lett. 14, 80 (1987).

${ }^{6}$ J. Yang and S. Epstein, Geochim. Cosmochim. Acta 51, 2011 (1987).

${ }^{7}$ J. Yang and S. Epstein, Geochim. Cosmochim. Acta 51, 2019 (1987).

${ }^{8}$ M. H. Thiemens and T. Jackson, Geophys. Res. Lett. 14, 624 (1987).

${ }^{9}$ M. H. Thiemens and T. Jackson, Geophys. Res. Lett. 15, 639 (1988).

${ }^{10}$ S. M. Anderson, J. Morton, and K. Mauersberger, Chem. Phys. Lett. 156, 175 (1989)

${ }^{11}$ J. Morton, B. Schueler, and K. Mauersberger, Chem. Phys. Lett. 154, 143 (1989).

${ }^{12}$ J. Morton, J. Barnes, B. Schueler, and K. Mauersberger, J. Geophys. Res. 95, 901 (1990)

${ }^{13}$ M. H. Thiemens and T. Jackson, Geophys. Res. Lett. 17, 717 (1990).

${ }^{14}$ B. Schueler, J. Morton, and K. Mauersberger, Geophys. Res. Lett. 17, 1295 (1990)

${ }^{15}$ J. Wen and M. H. Thiemens, Chem. Phys. Lett. 172, 416 (1990).

${ }^{16}$ J. Wen and M. H. Thiemens, J. Geophys. Res. 96, 10911 (1991).

${ }^{17}$ S. M. Anderson, K. Mauersberger, J. Morton, and B. Schueler, ACS Symp. Ser. 502, 1 (1992).

${ }^{18}$ M. H. Thiemens, ACS Symp. Ser. 502, 1 (1992)

${ }^{19}$ K. Mauersberger, J. Morton, B. Schueler, and J. Stehr, Geophys. Res. Lett. 20, 1031 (1993).
${ }^{20}$ D. Krankowsky, F. Bartecki, G. G. Klees, K. Mauersberger, and K. Schellenbach, Geophys. Res. Lett. 22, 1713 (1995).

${ }^{21}$ J. Sehested, O. J. Nielsen, H. Egsgaard, N. W. Larsen, T. Pedersen, L. K. Christensen, and M. Wiegell, J. Geophys. Res. 100, 20979 (1995).

${ }^{22}$ D. Krankowsky and K. Mauersberger, Science 274, 1324 (1996).

${ }^{23}$ L. K. Christensen, N. W. Larsen, F. M. Nicolaisen, T. Pedersen, G. O. Sørensen, and H. Egsgaard, J. Mol. Spectrosc. 175, 220 (1996).

${ }^{24}$ J. C. Johnson and M. H. Thiemens, J. Geophys. Res. 102, 25395 (1997).

${ }^{25}$ S. M. Anderson, D. Hüsebusch, and K. Mauersberger, J. Chem. Phys. 107, 5385 (1997)

${ }^{26}$ K. Mauersberger, B. Erbacher, D. Krankowsky, J. Günther, and R. Nickel, Science 283, 370 (1999).

${ }^{27}$ C. Janssen, J. Günther, D. Krankowsky, and K. Mauersberger, J. Chem. Phys. 111, 7179 (1999).

${ }^{28}$ J. Günther, D. Krankowsky, and K. Mauersberger, Chem. Phys. Lett. 324, 31 (2000).

${ }^{29}$ M. H. Thiemens, Science 283, 341 (1999), and references cited therein.

${ }^{30}$ J. Farquhar, H. M. Bao, and M. H. Thiemens, Science 289, 756 (2000).

${ }^{31}$ H. Bao, M. H. Thiemens, J. Farquhar, D. A. Campbell, C. C.-W. Lee, K. Heine, and D. B. Loope, Nature (London) 406, 176 (2000).

${ }^{32}$ H. Hippler, R. Rahn, and J. Troe, J. Chem. Phys. 93, 6560 (1990).

${ }^{33}$ J. I. Steinfeld, S. M. Alder-Golden, and J. W. Gallagher, J. Phys. Chem. Ref. Data 16, 911 (1987), and references cited therein.

${ }^{34}$ M. R. Wiegell, N. W. Larsen, T. Pedersen, and H. Egsgaard, Int. J. Chem. Kinet. 29, 745 (1997).

${ }^{35}$ S. M. Anderson, F. S. Klein, and F. Kaufman, J. Chem. Phys. 83, 1648 (1985)

${ }^{36}$ P. Pechukas and J. C. Light, J. Chem. Phys. 42, 3281 (1965).

${ }^{37}$ P. Pechukas, R. Rankin, and J. C. Light, J. Chem. Phys. 44, 794 (1966).

${ }^{38}$ T. Baer and W. L. Hase, Unimolecular Reaction Dynamics, Theory and Experiment (Oxford University Press, New York, 1996), and references cited therein

${ }^{39}$ R. G. Gilbert and S. C. Smith, Theory of Unimolecular and Recombination Reactions (Blackwell Scientific, Boston, 1990), and references cited therein.

${ }^{40}$ R. N. Zare, Angular Momentum: Understanding Spatial Aspects in Chemistry and Physics (Wiley, New York, 1988), and references cited therein.

${ }^{41}$ E. Gorin, Acta Physicochim. URSS 9, 691 (1938).

${ }^{42}$ E. Gorin, W. Kauzmann, J. Walter, and H. Eyring, J. Chem. Phys. 7, 633 (1939)

${ }^{43}$ S. Glasstone, K. J. Laidler, and H. Eyring, The Theory of Rate Processes (McGraw-Hill, New York, 1941), p. 260.

${ }^{44}$ D. A. McQuarrie, Statistical Mechanics (Harper \& Row, New York, 1976), and references cited therein.

${ }^{45}$ P. J. Robinson and K. A. Holbrook, Unimolecular Reactions (WileyInterscience, London, 1972), and references cited therein.

${ }^{46} \mathrm{~W}$. Forst, Theory of Unimolecular Reactions (Academic, New York, 1973), and references cited therein.

${ }^{47}$ The uncertainty relation between energy and lifetime is $\tau \Delta E \geqslant \hbar$ [A. Messiah, Quantum Mechanics (North-Holland, New York, 1966), Vol. 1]. For $\tau=3 \mathrm{ps}$ one finds $\Delta E \approx 100 / 18 \pi \approx 1 \mathrm{~cm}^{-1}$.

${ }^{48}$ T. Beyer and D. F. Swinehart, Commun. ACM 16, 379 (1973).

${ }^{49}$ T. L. Hill, An Introduction to Statistical Thermodynamics (Dover, New York, 1986), and references cited therein.

${ }^{50}$ J. A. Kaye, J. Geophys. Res. 91, 7865 (1986). The number of significant figures for $\sigma$ and $\epsilon$ is, of course, fewer than cited in the line preceding the present Eq. (4.14).

${ }^{51}$ R. C. Reid, J. M. Prausnitz, and T. K. Sherwood, The Properties of Gases and Liquids, 3rd ed. (McGraw-Hill, New York, 1977). The number of significant figures in the expression for $\Omega_{2,2}^{*}$ is, of course, fewer than those cited in the quoted expression for the collision integral, Eq. (4.15).

${ }^{52}$ G. Herzberg, Molecular Spectra and Molecular Structure, Vol. 2, Infrared and Raman Spectra of Polyatomic Molecules (Van Nostrand Reinhold, New York, 1945).

${ }^{53}$ S. V. Ivanov and V. Y. Panchenko, Phys. Usp. 37, 677 (1994), and references cited therein.

${ }^{54}$ J. M. Flaud and R. Bacis, Spectrochim. Acta 54, 3 (1998), and references cited therein

${ }^{55}$ These rate constants differ slightly from those in Ref. 25 , where in the definition of the rate constant an extra factor of 2 was added. In the present case, we have defined the rate constant so as not to include the factor of 2, in conformity with Ref. 26.

${ }^{56}$ W. L. Hase, J. Chem. Phys. 64, 2442 (1976).

${ }^{57}$ M. Quack and J. Troe, Ber. Bunsenges. Phys. Chem. 81, 329 (1977). 
${ }^{58}$ G. P. Smith and D. M. Golden, Int. J. Chem. Kinet. 10, 489 (1978).

${ }^{59}$ S. N. Rai and D. G. Truhlar, Int. J. Chem. Kinet. 79, 6046 (1983).

${ }^{60} \mathrm{~S}$. W. Benson, Can. J. Chem. 61, 881 (1983).

${ }^{61}$ W. L. Hase and D. M. Wardlaw, in Bimolecular Collisions, edited by J. E. Baggott and M. N. Ashfold (Royal Society of Chemistry, Burlington House, London, 1989), p. 171.

${ }^{62}$ X. Hu and W. L. Hase, J. Phys. Chem. 93, 6029 (1989).
${ }^{63}$ D. M. Wardlaw and R. A. Marcus, Adv. Chem. Phys. 70, 231 (1988).

${ }^{64}$ S. J. Klippenstein and R. A. Marcus, J. Chem. Phys. 91, 2280 (1989).

${ }^{65}$ S. J. Klippenstein and R. A. Marcus, J. Chem. Phys. 93, 2418 (1990).

${ }^{66}$ S. J. Klippenstein, J. Chem. Phys. 94, 6469 (1991).

${ }^{67}$ S. J. Klippenstein and Y.-W. Kim, J. Chem. Phys. 99, 5790 (1993).

${ }^{68} \mathrm{~S}$. J. Klippenstein, in The Chemical Dynamics and Kinetics of Small Radicals, Part I, edited by K. Liu and A. Wagner (World Scientific, Singapore, 1995), and references cited therein. 